Göteborg

ITP 94-40

hep-th/9412188

December 1994

\title{
Fermionic and bosonic pair creation in an external electric field at finite temperature using the functional Schrödinger representation
}

\author{
Joakim Hallin円 and Per Liljenberg' \\ Institute of Theoretical Physics \\ Chalmers University of Technology \\ and University of Göteborg \\ S-412 96 Göteborg, Sweden
}

\begin{abstract}
We solve the time evolution of the density matrix both for fermions and bosons in the presence of a homogeneous but time dependent external electric field. The number of particles produced by the external field, as well as their distribution in momentum space is found for finite times. Furthermore, we calculate the probability of finding a given number of particles in the ensemble. In all cases, there is a nonvanishing thermal contribution. The bosonic and the fermionic density matrices are expressed in a "functional field basis". This constitutes an extension of the "field basis" concept to fermions.
\end{abstract}

\footnotetext{
${ }^{1}$ Email address: tfejh@fy.chalmers.se

${ }^{2}$ Email address: tfepl@fy.chalmers.se
} 


\section{Introduction}

One of the characteristic features of quantum field theory is the non-conservation of the number of particles. The most familiar cases are the collision processes, where genuinely quantum mechanical interactions lead to creation (or annihilation) of particles. Here, all the interacting fields are quantum fields. It has been known for long that also classical external fields can change the number of particles. This is the well-known Schwinger mechanism for particle production in an external field [1]. The Schwinger formula has been derived in a number of ways and has found a wide range of applications, from Hawking radiation [2] to heavy ion collisions [3].

Schwinger's result has some shortcomings. The external field is homogeneous throughout space; the timescale is large (infinite) and it applies only at zero temperature. Furthermore the probability of finding the system in the groundstate after some time has elapsed is not related in a simple manner to observable quantities, like the average number of particles created because the probability distribution is non-trivial.

The first problem has to some extent been discussed elsewhere [4] and will not be considered here.

The problem of large times comes about because of the nature of the model, which consists of free particles minimally coupled to an external background field. In this case the backreaction, i.e. the field generated by created particles is neglected. Since the number of particles, and hence the induced field, increases with time there is a maximal time for which the background field approximation applies. If one insists on that approximation, which we do, it is important to keep the explicit time dependence throughout the calculations. The backreaction has been studied extensively in [5].

The third problem of extending the pair creation phenomenon to non-zero temperatures has been studied in thermal field theory, where the effective action is a natural object of interest. However, there seems to be confusion about the influence of a thermal background on the pair creation [6, 7, 8]. In the real time formalism one does not find a thermal correction whereas using imaginary time there appears to be one. It is also not clear if any of the formalisms actually are applicable to this kind of problems. In this context it is again necessary to keep the time dependence and care must be taken with the infinite time limit. We find that at fixed temperature, the thermal contribution to pair production will vanish in the infinite time limit. At any finite time, however, there is a significant thermal effect. In the background field approximation, one can therefore question the use of effective lagrangians (the infinite time limit being understood) at least concerning the imaginary part responsible for pair production.

So much for the physics, we now turn to the formalism. We will be working in the functional Schrödinger representation (see e.g. [9, 10, 11]) which is convenient for many reasons. Because of the close analogy with quantum mechanics, there are no conceptual problems in this picture. We do not suffer from doubts about the formalism and/or applicability, as in thermal field theory. Furthermore, the treatment of time and temperature is straightforward.

In order to make the calculations explicit we make extensive use of the so called "field 
basis" concept, i.e. one views the wave functionals as overlaps of a general state and a "field basis state". This is known to be possible for bosons. We extend the formalism to fermions. One then has to be careful with the Grassmann nature of the basis. Also extra care must be taken when working in a reducible representation, which is the case at hand.

With this setup, the strategy is simple. We solve the time evolution for the thermal density matrix of the system under consideration, i.e. we find $\rho$ satisfying

$$
i \dot{\rho}=[H, \rho], \quad \rho(0)=\text { equilibrium ensemble. }
$$

Then we simply calculate the thermal average of the number operator $N$, that is

$$
\langle N\rangle=\operatorname{tr}[N \rho(t)]
$$

Closely related to this quantity is the number density in momentum space (transverse and longitudinal momenta). We also find the probability distribution of the n-particle states in the ensemble at arbitrary times, specifically we calculate the probability of finding the system in the groundstate at finite times. The paper consists of two main sections. In the first section we treat bosons and set up the machinery. Fermions are dealt with in the second section. After a careful discussion of the fermionic field basis, all the calculations previously done for bosons are repeated for fermions. The paper ends with a few concluding remarks.

A word of caution; throughout this paper there are numerous seemingly infinite quantities. This is not case however, since we always work with a UV-cutoff. Only in the expressions that are not only convergent but also quite insensitive to the cutoff, do we remove this cutoff.

\section{Bosons}

The hamiltonian for a charged scalar field in an external electromagnetic field is

$$
\hat{H}=\int d^{3} x \hat{\pi}(\mathbf{x}) \hat{\pi}^{\dagger}(\mathbf{x})+\int d^{3} x d^{3} y \hat{\phi}^{\dagger}(\mathbf{x}) \omega^{2}(\mathbf{x}, \mathbf{y}) \hat{\phi}(\mathbf{y})
$$

where

$$
\omega^{2}(\mathbf{x}, \mathbf{y})=\left(-\left(\nabla_{x}-i e \mathbf{A}(\mathbf{x})\right)^{2}+m^{2}\right) \delta(\mathbf{x}-\mathbf{y})
$$

and we use the convention that $e=-|e|<0$. In a standard manner [9, 10] we represent the field operators and the states:

$$
\begin{array}{cl}
\hat{\phi} \leftrightarrow \phi, \hat{\pi} \leftrightarrow \frac{1}{i} \frac{\delta}{\delta \phi} \quad, & \hat{\phi}^{\dagger} \leftrightarrow \phi^{*}, \hat{\pi}^{\dagger} \leftrightarrow \frac{1}{i} \frac{\delta}{\delta \phi^{*}} \\
|\Psi\rangle \leftrightarrow \Psi\left(\phi, \phi^{*}\right) & , \quad\langle\Psi| \leftrightarrow \Psi\left(\phi, \phi^{*}\right)^{*} .
\end{array}
$$

$\mathrm{By} *$ we mean complex conjugation. The inner product is defined by functional integration

$$
\langle\Psi \mid \Phi\rangle=\int \mathcal{D}^{2} \phi \Psi\left(\phi, \phi^{*}\right)^{*} \Phi\left(\phi, \phi^{*}\right),
$$


where $\mathcal{D}^{2} \phi=\mathcal{D} \phi \mathcal{D} \phi^{*}$. As a reference we give a formula for gaussian functional integrals:

$$
\begin{aligned}
& \int \mathcal{D}^{2} \phi \exp \left[-\int d^{3} x d^{3} y \phi^{*} \Omega \phi+\int d^{3} x\left(a^{*} \phi+\phi^{*} b\right)\right]= \\
&=\operatorname{det}(\Omega)^{-1} \exp \left[\int d^{3} x d^{3} y a^{*} \Omega^{-1} b\right],
\end{aligned}
$$

where the determinant is defined through $\operatorname{det} \Omega=\exp \operatorname{Tr} \ln \Omega$ with a functional logarithm and $\operatorname{Tr} \Omega=\int d^{3} x \Omega(\mathbf{x}, \mathbf{x})$.

The state functional can be viewed as an overlap between a general state and a "field basis state", thus

$$
\Psi\left(\phi, \phi^{*}\right)=\left\langle\phi, \phi^{*} \mid \Psi\right\rangle
$$

The field basis has the following properties [9]:

$$
\begin{aligned}
\hat{\phi}\left|\phi, \phi^{*}\right\rangle & =\phi\left|\phi, \phi^{*}\right\rangle, \\
\left\langle\phi, \phi^{*} \mid \varphi, \varphi^{*}\right\rangle & =\delta(\phi-\varphi) \delta\left(\phi^{*}-\varphi^{*}\right), \\
\hat{1} & =\int \mathcal{D}^{2} \phi\left|\phi, \phi^{*}\right\rangle\left\langle\phi, \phi^{*}\right| .
\end{aligned}
$$

For operators we have

$$
\begin{aligned}
\left\langle\phi, \phi^{*}|\hat{\mathcal{O}}| \varphi, \varphi^{*}\right\rangle & =\mathcal{O}\left(\phi \phi^{*}, \varphi \varphi^{*}\right) \\
\operatorname{tr} \hat{\mathcal{O}} & =\int \mathcal{D}^{2} \phi\left\langle\phi, \phi^{*}|\hat{\mathcal{O}}| \phi, \phi^{*}\right\rangle \\
\left\langle\phi, \phi^{*}\left|\hat{\mathcal{O}}\left(\hat{\phi}, \hat{\phi}^{\dagger}, \hat{\pi}, \hat{\pi}^{\dagger}\right)\right| \Psi\right\rangle & =\hat{\mathcal{O}}\left(\phi, \phi^{*}, \frac{\delta}{\delta \phi}, \frac{\delta}{\delta \phi^{*}}\right)\left\langle\phi, \phi^{*} \mid \Psi\right\rangle .
\end{aligned}
$$

\subsection{The initial ensemble}

The system under consideration is described by a density matrix $\rho(t)$. For a good introduction to density matrices see [12]. We intend to evolve the system in time, using the hamiltonian (3). The system evolves from an initial ensemble at thermal equilibrium at $t=0$ to a final ensemble at $t=t_{f}$ when the external field is switched off and the number of particles in the system is counted.

Initially the system is in thermal equilibrium and is described by an unnormalized stationary density matrix $\hat{\rho}_{u}=e^{-\beta \hat{H}_{0}}$ satisfying

$$
-\partial_{\beta} \hat{\rho}_{u}=\hat{H}_{0} \hat{\rho}_{u}
$$

where $\hat{H}_{0}$ is the hamiltonian (3) with $\mathbf{A}=0$ and $\beta$ is the inverse temperature, $\beta=\frac{1}{k_{B} T}$. Note that such an ensemble cannot be created by pure QED since it contains all possible charges. Furthermore we have

$$
\lim _{\beta \rightarrow 0} \hat{\rho}_{u}=\hat{1} \quad, \quad \hat{\rho}_{u}^{\dagger}=\hat{\rho}_{u}
$$


In the functional representation $\hat{\rho}_{u}$ is represented by a functional matrix $\rho_{u}\left(\phi_{1} \phi_{1}^{*}, \phi_{2} \phi_{2}^{*}\right)=$ $\left\langle\phi_{1}, \phi_{1}^{*}\left|\hat{\rho}_{u}\right| \phi_{2}, \phi_{2}^{*}\right\rangle$, thus

$$
-\partial_{\beta} \rho_{u}\left(\phi_{1} \phi_{1}^{*}, \phi_{2} \phi_{2}^{*}\right)=\hat{H}_{0}\left(\phi_{1}, \phi_{1}^{*}, \frac{\delta}{\delta \phi_{1}}, \frac{\delta}{\delta \phi_{1}^{*}}\right) \rho_{u}\left(\phi_{1} \phi_{1}^{*}, \phi_{2} \phi_{2}^{*}\right)
$$

and the two additional conditions read:

$$
\begin{aligned}
\lim _{\beta \rightarrow 0} \rho_{u}\left(\phi_{1} \phi_{1}^{*}, \phi_{2} \phi_{2}^{*}\right) & =\delta\left(\phi_{1}-\phi_{2}\right) \delta\left(\phi_{1}^{*}-\phi_{2}^{*}\right) \\
\rho_{u}\left(\phi_{1} \phi_{1}^{*}, \phi_{2} \phi_{2}^{*}\right) & =\rho_{u}^{*}\left(\phi_{2} \phi_{2}^{*}, \phi_{1} \phi_{1}^{*}\right) .
\end{aligned}
$$

In order to solve for $\rho_{u}$, we make a gaussian ansatz with a covariance $\Omega_{i j}^{0}$

$$
\rho_{u}\left(\phi_{1} \phi_{1}^{*}, \phi_{2} \phi_{2}^{*}\right)=N_{u} \exp \left[-\int d^{3} x d^{3} y \phi_{i}^{*}(\mathbf{x}) \Omega_{i j}^{0}(\mathbf{x}, \mathbf{y}) \phi_{j}(\mathbf{y})\right] \quad i, j=1,2 .
$$

One obtains

$$
\begin{aligned}
-\partial_{\beta} \ln N_{u} & =\operatorname{Tr} \Omega_{11}^{0} \\
-\partial_{\beta} \Omega_{11}^{0} & =\Omega_{11}^{0}{ }^{2}-\omega_{0}^{2} \\
-\partial_{\beta} \Omega_{12}^{0} & =\Omega_{11}^{0} \Omega_{12}^{0} \\
-\partial_{\beta} \Omega_{21}^{0} & =\Omega_{21}^{0} \Omega_{11}^{0} \\
-\partial_{\beta} \Omega_{22}^{0} & =\Omega_{21}^{0} \Omega_{12}^{0}
\end{aligned}
$$

where $\omega_{0}=\omega$ having set $\mathbf{A}=0$. We have also used the notation

$$
(\Omega \tilde{\Omega})(\mathbf{x}, \mathbf{y})=\int d^{3} z \Omega(\mathbf{x}, \mathbf{z}) \tilde{\Omega}(\mathbf{z}, \mathbf{y})
$$

Due to translational invariance the covariances will satisfy $\Omega_{i j}^{0}(\mathbf{x}, \mathbf{y})=\Omega_{i j}^{0}(\mathbf{x}-\mathbf{y})$. We can therefore simplify the equations by performing a fourier transform according to

$$
\phi(\mathbf{x})=\int_{p} \phi(\mathbf{p}) e^{i \mathbf{p} \cdot \mathbf{x}}, \pi(\mathbf{x})=\int_{p} \pi(\mathbf{p}) e^{-i \mathbf{p} \cdot \mathbf{x}}, \Omega(\mathbf{x}-\mathbf{y})=\int_{p} \Omega(\mathbf{p}) e^{i \mathbf{p} \cdot(\mathbf{x}-\mathbf{y})}
$$

where $\int_{p}=\int d^{3} \mathbf{p} /(2 \pi)^{3}$. The equations then read

$$
\begin{aligned}
-\partial_{\beta} \ln N_{u} & =V \int_{p} \Omega_{11}^{0}(\mathbf{p}) \\
-\partial_{\beta} \Omega_{11}^{0}(\mathbf{p}) & =\Omega_{11}^{0}(\mathbf{p})^{2}-\omega_{0}(\mathbf{p})^{2} \\
-\partial_{\beta} \Omega_{12}^{0}(\mathbf{p}) & =\Omega_{11}^{0}(\mathbf{p}) \Omega_{12}^{0}(\mathbf{p}) \\
-\partial_{\beta} \Omega_{21}^{0}(\mathbf{p}) & =\Omega_{21}^{0}(\mathbf{p}) \Omega_{11}^{0}(\mathbf{p}) \\
-\partial_{\beta} \Omega_{22}^{0}(\mathbf{p}) & =\Omega_{21}^{0}(\mathbf{p}) \Omega_{12}^{0}(\mathbf{p})
\end{aligned}
$$

where $\omega_{0}(\mathbf{p})=\sqrt{\mathbf{p}^{2}+m^{2}}$ and $V=(2 \pi)^{3} \delta^{3}(0)$ is the large but finite spatial volume. The hermiticity conditions on $\rho_{u}$ yield

$$
\Omega_{11}^{0}(\mathbf{p})^{*}=\Omega_{22}^{0}(\mathbf{p}), \Omega_{12}^{0}(\mathbf{p})^{*}=\Omega_{12}^{0}(\mathbf{p}), \Omega_{21}^{0}(\mathbf{p})^{*}=\Omega_{21}^{0}(\mathbf{p})
$$


The solution to (29)-(34) leading to a density matrix that satisfies the delta function condition (19) is

$$
\begin{aligned}
\Omega_{11}^{0}(\mathbf{p}) & =\Omega_{22}^{0}(\mathbf{p})=\omega_{0} \operatorname{coth} \beta \omega_{0} \\
\Omega_{12}^{0}(\mathbf{p}) & =\Omega_{21}^{0}(\mathbf{p})=-\frac{\omega_{0}}{\sinh \beta \omega_{0}} \\
N_{u} & =\exp \left[V \int_{p} \ln \frac{\omega_{0}}{\sinh \beta \omega_{0}}\right]
\end{aligned}
$$

The precise value of $N_{u}$ can be found using $\lim _{\beta \rightarrow 0} \int \mathcal{D}^{2} \phi_{1} \rho_{u}\left(\phi_{1} \phi_{1}^{*}, \phi_{2} \phi_{2}^{*}\right)=1$ which follows from (19). The partition function $Z=\operatorname{tr} \hat{\rho}_{u}$ and the normalized density matrix $\rho_{0}=\rho_{u} / \operatorname{tr} \hat{\rho}_{u}$ are now readily found as

$$
\begin{gathered}
Z=\exp \left[-V \int_{p} 2 \ln \left(2 \sinh \left(\frac{\beta \omega_{0}}{2}\right)\right)\right] \\
\rho_{0}\left(\phi_{1} \phi_{1}^{*}, \phi_{2} \phi_{2}^{*}\right)=\exp \left[V \int_{p} \ln \left(2 \omega_{0} \tanh \frac{\beta \omega_{0}}{2}\right)\right] \times \\
\exp \left[-\int_{p}\left(\left(\phi_{1}^{*} \phi_{1}+\phi_{2}^{*} \phi_{2}\right) \omega_{0} \operatorname{coth} \beta \omega_{0}-\frac{\omega_{0}}{\sinh \beta \omega_{0}}\left(\phi_{1}^{*} \phi_{2}+\phi_{2}^{*} \phi_{1}\right)\right)\right]
\end{gathered}
$$

\subsection{The time dependent ensemble}

We solve the Liouville equation for the density matrix $\rho(t)$ in an external field $\mathbf{A}(t)$ with the initial condition specified in the previous section, i.e.

$$
\begin{aligned}
i \partial_{t} \hat{\rho}(t) & =[\hat{H}(t), \hat{\rho}(t)] \\
\hat{\rho}(0) & =\hat{\rho}_{0} .
\end{aligned}
$$

The external field is constant in space but varies in time as

$$
\mathbf{E}(t)=\left\{\begin{array}{cl}
0 & , t<0 \\
E \mathbf{e}_{3} & , 0<t<t_{f} \\
0 & , t_{f}<t
\end{array} \quad \mathbf{A}(t)=\left\{\begin{array}{cl}
0 & , t<0 \\
-E t \mathbf{e}_{3} & , 0<t<t_{f} \\
-E t_{f} \mathbf{e}_{3} & , t_{f}<t
\end{array}\right.\right.
$$

where $E>0$ so that $e E<0$. In the functional representation we find the equation for $\rho\left(\phi_{1} \phi_{1}^{*}, \phi_{2} \phi_{2}^{*}\right)$ :

$$
i \partial_{t} \rho\left(\phi_{1} \phi_{1}^{*}, \phi_{2} \phi_{2}^{*}\right)=\hat{H}\left(\phi_{1}, \phi_{1}^{*}, \frac{\delta}{\delta \phi_{1}}, \frac{\delta}{\delta \phi_{1}^{*}}\right) \rho\left(\phi_{1} \phi_{1}^{*}, \phi_{2} \phi_{2}^{*}\right)-\hat{H}\left(\phi_{2}, \phi_{2}^{*}, \frac{\delta}{\delta \phi_{2}}, \frac{\delta}{\delta \phi_{2}^{*}}\right) \rho\left(\phi_{1} \phi_{1}^{*}, \phi_{2} \phi_{2}^{*}\right)
$$

Again we make a gaussian ansatz,

$$
\rho\left(\phi_{1} \phi_{1}^{*}, \phi_{2} \phi_{2}^{*}, t\right)=N(t) \exp \left[-\int d^{3} x d^{3} y \phi_{i}^{*}(\mathbf{x}) \Omega_{i j}(\mathbf{x}, \mathbf{y}, t) \phi_{j}(\mathbf{y})\right]
$$


and perform a fourier transform as in the previos case (with the specified external field the hamiltonian is still translationally invariant). Suppressing the time and momentum dependence, the equations obtained are

$$
\begin{aligned}
i \partial_{t} \ln N & =V \int_{p}\left(\Omega_{11}-\Omega_{22}\right) \\
i \partial_{t} \Omega_{11} & =\Omega_{11}{ }^{2}-\omega^{2}-\Omega_{12} \Omega_{21} \\
i \partial_{t} \Omega_{12} & =\Omega_{12}\left(\Omega_{11}-\Omega_{22}\right) \\
i \partial_{t} \Omega_{21} & =\Omega_{21}\left(\Omega_{11}-\Omega_{22}\right) \\
i \partial_{t} \Omega_{22} & =-\Omega_{22}{ }^{2}+\omega^{2}-\Omega_{12} \Omega_{21}
\end{aligned}
$$

where $\omega(\mathbf{p})=\sqrt{(\mathbf{p}-e \mathbf{A})^{2}+m^{2}}$. The density matrix $\rho$ is hermitian and the hermiticity conditions read

$$
\Omega_{11}^{*}=\Omega_{22}, \Omega_{12}{ }^{*}=\Omega_{12}, \Omega_{21}^{*}=\Omega_{21}
$$

By inspection one finds that the solution to three of the equations are

$$
\begin{aligned}
& \Omega_{12}=a\left(\Omega_{11}+\Omega_{22}\right) \\
& \Omega_{21}=b\left(\Omega_{11}+\Omega_{22}\right) \\
& \Omega_{22}=\Omega_{11}{ }^{*}
\end{aligned}
$$

where $a$ and $b$ are constants of motion determined by the initial conditions. From (35) and (36) we find $a=b=-1 / 2 \cosh \beta \omega_{0}$. Eliminating $\Omega_{12}, \Omega_{21}$ and $\Omega_{22}$ we find the remaining two equations

$$
\begin{aligned}
i \partial_{t} \ln N & =V \int_{p}\left(\Omega-\Omega^{*}\right) \\
i \partial_{t} \Omega & =\Omega^{2}-\omega^{2}-\frac{1}{4 \cosh ^{2} \beta \omega_{0}}\left(\Omega+\Omega^{*}\right)^{2}
\end{aligned}
$$

where we have set $\Omega_{11}=\Omega$. Hence the problem has been reduced to finding $\Omega$. Now write

$$
\Omega=-i \frac{\partial_{t} y}{y} \quad y=r \exp \left(i \theta \operatorname{coth} \beta \omega_{0}\right)
$$

Inserting these expressions into (55) and rewriting the initial condition, (35), we find

$$
\begin{aligned}
\ddot{r}-\frac{\bar{\omega}_{0}^{2}}{r^{3}}+\bar{\omega}^{2} r & =0 \quad, \quad r(0)=1 \quad \dot{r}(0)=0 \\
r^{2} \dot{\theta} & =\bar{\omega}_{0} \quad, \quad \theta(0)=0 \quad \dot{\theta}(0)=\bar{\omega}_{0}
\end{aligned}
$$

Here we have introduced dimensionless quantities

$$
\begin{aligned}
& \bar{t}=t \sqrt{|e E|}, \quad \overline{\mathbf{p}}=\mathbf{p} / \sqrt{|e E|}, \quad \bar{m}=m / \sqrt{|e E|}, \quad \bar{\beta}=\beta \sqrt{|e E|}, \\
& \Lambda=\left(\bar{p}^{1}\right)^{2}+\left(\bar{p}^{2}\right)^{2}+\bar{m}^{2}, \quad \bar{\omega}=\sqrt{\Lambda+\left(\bar{p}^{3}-\bar{t}\right)^{2}}, \quad \bar{V}=V|e E|^{3 / 2}
\end{aligned}
$$


and the overdots in (57) denote $\partial / \partial \bar{t}$. Finally introducing the complex function $z=r \exp (i \theta)$ the set of equations (57) can be collected into the form

$$
\begin{aligned}
\ddot{z}+\bar{\omega}^{2} z & =0 \\
z(0) & =1 \\
\dot{z}(0) & =i \bar{\omega}_{0}
\end{aligned}
$$

One can now find a closed expression for $\Omega$ in terms of special functions, since the general solution of (59) is a linear combination of two parabolic cylinder functions [13]

$$
D_{-\frac{i}{2} \Lambda-\frac{1}{2}}\left( \pm(1+i)\left(\bar{t}-\bar{p}^{3}\right)\right) .
$$

The solution satisfying the initial conditions is easily written down but since the expression is rather long we don't give it here. Instead we end this section with the expression for the time dependent normalized density matrix,

$$
\begin{aligned}
& \rho(t)=\exp \left[V \int_{p} \ln \left(\left(\Omega+\Omega^{*}\right) \tanh \beta \omega_{0} \tanh \frac{\beta \omega_{0}}{2}\right)\right] \times \\
& \exp \left[-\int_{p}\left(\phi_{1}^{*} \Omega \phi_{1}-\left(\phi_{1}^{*} \phi_{2}+\phi_{1}^{*} \phi_{2}\right)\left(\Omega+\Omega^{*}\right) \frac{1}{2 \cosh \beta \omega_{0}}+\phi_{2}^{*} \Omega^{*} \phi_{2}\right)\right],
\end{aligned}
$$

where $\Omega$ in terms of $r=|z|$ reads

$$
\Omega(t)=\sqrt{|e E|}\left(\frac{\bar{\omega}_{0} \operatorname{coth} \bar{\beta} \bar{\omega}_{0}}{r^{2}}-i \frac{\dot{r}}{r}\right) .
$$

\subsection{Expectation value of the number operator}

Once the density matrix has been found one can start asking questions about the system. We will be concerned with particle creation and therefore concentrate on thermal expectation values of the number operator,

$$
\begin{aligned}
\langle\hat{N}\rangle(t) & =\operatorname{tr}(\hat{N} \hat{\rho}(t)) \\
& =\int \mathcal{D}^{2} \phi_{1} \mathcal{D}^{2} \phi_{2} \delta\left(\phi_{1}-\phi_{2}\right) \delta\left(\phi_{1}^{*}-\phi_{2}^{*}\right) \hat{N}\left(\phi_{1}, \phi_{1}^{*}\right) \rho\left(\phi_{1} \phi_{1}^{*}, \phi_{2} \phi_{2}^{*} ; t\right) .
\end{aligned}
$$

More specifically we will calculate the number of particles at $t=t_{f}$ just after the field has been turned off. We then find ourselves in the gauge $\mathbf{A}=-E t_{f} \mathbf{e}_{3}$. In order to make use of operators expressed in terms of fields in the $\mathbf{A}=0$ gauge we make a transformation to that gauge. The fields transform according to

$$
\mathbf{A}(x) \rightarrow \mathbf{A}(x)-\nabla \lambda(x) \quad \phi(x) \rightarrow \phi(x) e^{-i e \lambda(x)} .
$$

Since the physical states are gauge invariant the covariance transforms as

$$
\Omega(x, y) \rightarrow \Omega^{g}(x, y)=\Omega(x, y) e^{-i e(\lambda(x)-\lambda(y))} .
$$


For the specific transformation that changes $\mathbf{A}=-E t_{f} \mathbf{e}_{3}$ into $\mathbf{A}=0$, the effect on the covariance in the density matrix (62) simply amounts to a shift in the momentum

$$
\mathbf{p} \rightarrow \mathbf{p}_{g}=\mathbf{p}+e \mathbf{A}\left(t_{f}\right)=\left(p^{1}, p^{2}, p^{3}-e E t_{f}\right)
$$

Hence $r, \omega$ and $\omega_{0}$ transform as follows

$$
\begin{aligned}
r(\mathbf{p}) & \rightarrow r_{g}(\mathbf{p})=r\left(\mathbf{p}_{g}\right) \\
\bar{\omega}(\mathbf{p}) & \rightarrow \bar{\omega}^{g}(\mathbf{p})=\sqrt{\Lambda+\left(\bar{p}^{3}-\bar{t}+\bar{t}_{f}\right)^{2}} \\
\bar{\omega}_{0}(\mathbf{p}) & \rightarrow \bar{\omega}_{0}^{g}(\mathbf{p})=\sqrt{\Lambda+\left(\bar{p}^{3}+\bar{t}_{f}\right)^{2}} .
\end{aligned}
$$

Now find the number operator $\hat{N}$. The gauge invariant stationary groundstate, expressed in terms of fields in the $\mathbf{A}=0$ gauge, is given by

$$
\Psi_{0}\left(\phi, \phi^{*}\right)=\operatorname{det}\left(\omega_{0}\right)^{-1 / 2} \exp \left[-\int_{p} \phi^{*} \omega_{0} \phi\right]
$$

Associated with this groundstate are the annihilation operators

$$
\begin{aligned}
& \hat{a}(\mathbf{p})=\frac{1}{\sqrt{2 \omega_{0}(\mathbf{p})}}\left(\omega_{0}(\mathbf{p}) \hat{\phi}(\mathbf{p})+i \hat{\pi}^{\dagger}(\mathbf{p})\right) \\
& \hat{b}(\mathbf{p})=\frac{1}{\sqrt{2 \omega_{0}(\mathbf{p})}}\left(\omega_{0}(\mathbf{p}) \hat{\phi}^{\dagger}(\mathbf{p})+i \hat{\pi}(\mathbf{p})\right) .
\end{aligned}
$$

The states created by $\hat{a}^{\dagger}(\mathbf{p})$ have charge $e$ and physical momentum $\mathbf{p}$, while states created by $\hat{b}^{\dagger}(\mathbf{p})$ have charge $(-e)$ and physical momentum $(-\mathbf{p})$, as seen from the momentum and charge operators expressed in terms of $a$ 's and $b$ 's,

$$
\begin{aligned}
& \hat{\mathbf{P}}=-\int d^{3} x\left(\nabla \hat{\phi} \hat{\pi}+\nabla \hat{\phi}^{\dagger} \hat{\pi}^{\dagger}\right)=\int_{p} \mathbf{p}\left(\hat{a}^{\dagger}(\mathbf{p}) \hat{a}(\mathbf{p})-\hat{b}^{\dagger}(\mathbf{p}) \hat{b}(\mathbf{p})\right) \\
& \hat{Q}=i e \int d^{3} x\left(\hat{\phi}^{\dagger} \hat{\pi}^{\dagger}-\hat{\phi} \hat{\pi}\right)=e \int_{p}\left(\hat{a}^{\dagger}(\mathbf{p}) \hat{a}(\mathbf{p})-\hat{b}^{\dagger}(\mathbf{p}) \hat{b}(\mathbf{p})\right) .
\end{aligned}
$$

The expression for the number operator in the $\mathbf{A}=0$ gauge is

$$
\begin{aligned}
\hat{N} & =\int_{p}\left(\hat{a}^{\dagger}(\mathbf{p}) \hat{a}(\mathbf{p})+\hat{b}^{\dagger}(\mathbf{p}) \hat{b}(\mathbf{p})\right) \\
& =\int_{p}\left(\frac{1}{\omega_{0}(\mathbf{p})} \hat{\pi}^{\dagger}(\mathbf{p}) \hat{\pi}(\mathbf{p})+\omega_{0}(\mathbf{p}) \hat{\phi}^{\dagger}(\mathbf{p}) \hat{\phi}(\mathbf{p})-V\right) .
\end{aligned}
$$

By (63) and the gauge transformed versions of (61) and (62), we find after some manipulations the result

$$
\langle\hat{N}\rangle\left(t_{f}\right)=\frac{V}{2} \int_{p}\left[\left(\frac{\bar{\omega}_{0}}{\bar{\omega}_{0}^{g}} r_{g}^{2}+\frac{1}{\bar{\omega}_{0} \bar{\omega}_{0}^{g}} \dot{r}_{g}^{2}+\frac{\bar{\omega}_{0}^{g}}{\bar{\omega}_{0}} r_{g}^{-2}\right) \operatorname{coth} \frac{\bar{\beta} \bar{\omega}_{0}^{g}}{2}-2\right] .
$$



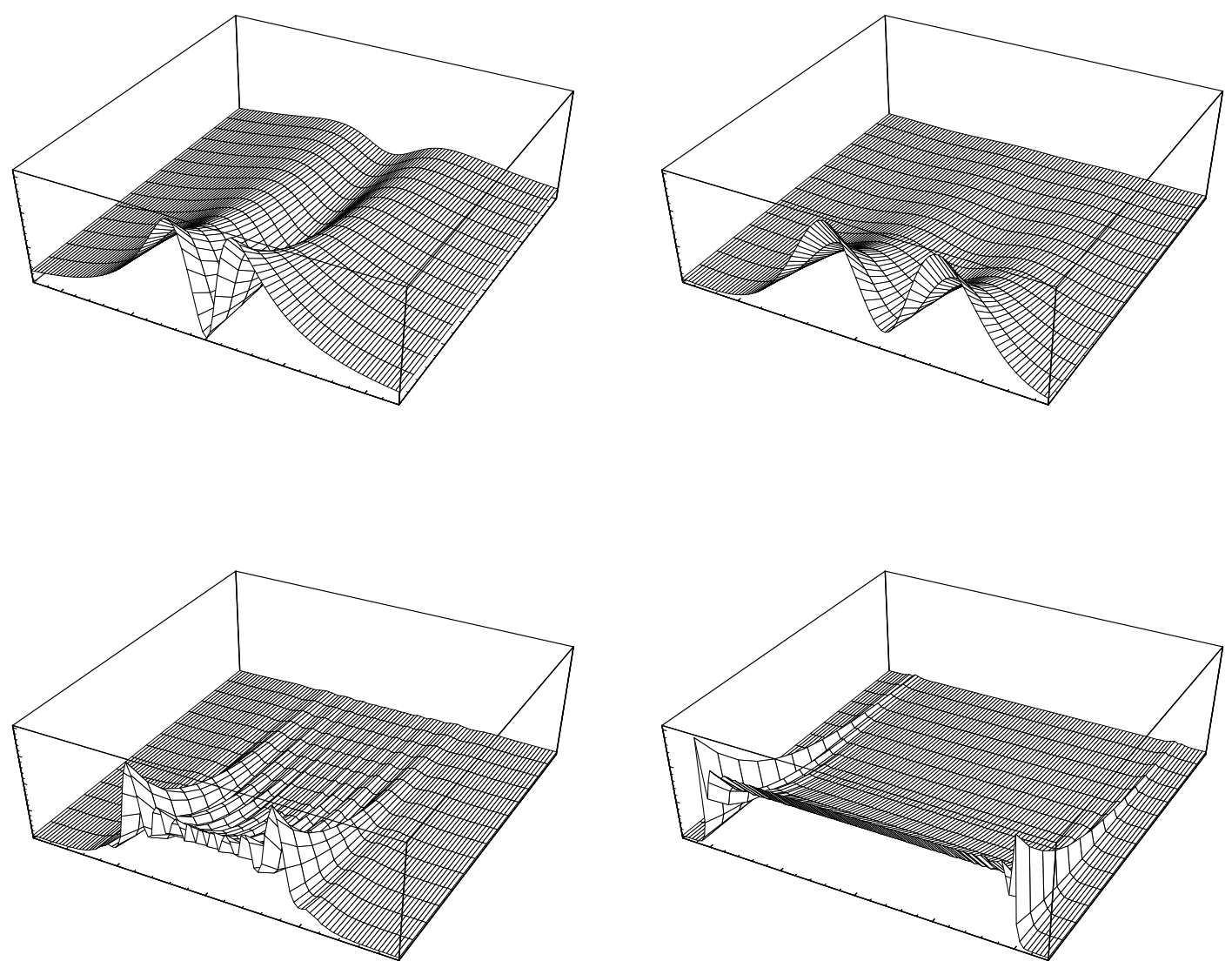

Figure 1: The momentum distribution, $n(-\mathbf{p}) / 2$, of the positively charged bosons at zero temperature. All quantities are expressed in units of the electric field.

To find the number of particles actually created during the process $\langle\hat{N}\rangle^{\text {cr }}$, we subtract the number of particles at $t=0$ :

$$
\left.\langle\hat{N}\rangle\right|_{t=0}=V \int_{p}\left[\operatorname{coth} \frac{\bar{\beta} \bar{\omega}_{0}}{2}-1\right] .
$$

Hence, we obtain

$$
\begin{aligned}
\langle\hat{N}\rangle^{\mathrm{cr}}\left(t_{f}\right) & =V \int_{p} n\left(\mathbf{p}, t_{f}\right) \operatorname{coth} \frac{\bar{\beta} \bar{\omega}_{0}^{g}}{2} \\
& =\frac{\bar{V}}{2(2 \pi)^{2}} \int_{-\infty}^{\infty} d \bar{p}^{3} \int_{\bar{m}^{2}}^{\infty} d \Lambda n\left(\Lambda, \bar{p}^{3}, \bar{t}_{f}\right) \operatorname{coth} \frac{\bar{\beta} \bar{\omega}_{0}^{g}}{2}
\end{aligned}
$$

where we have defined the zero temperature number density of the created particles 


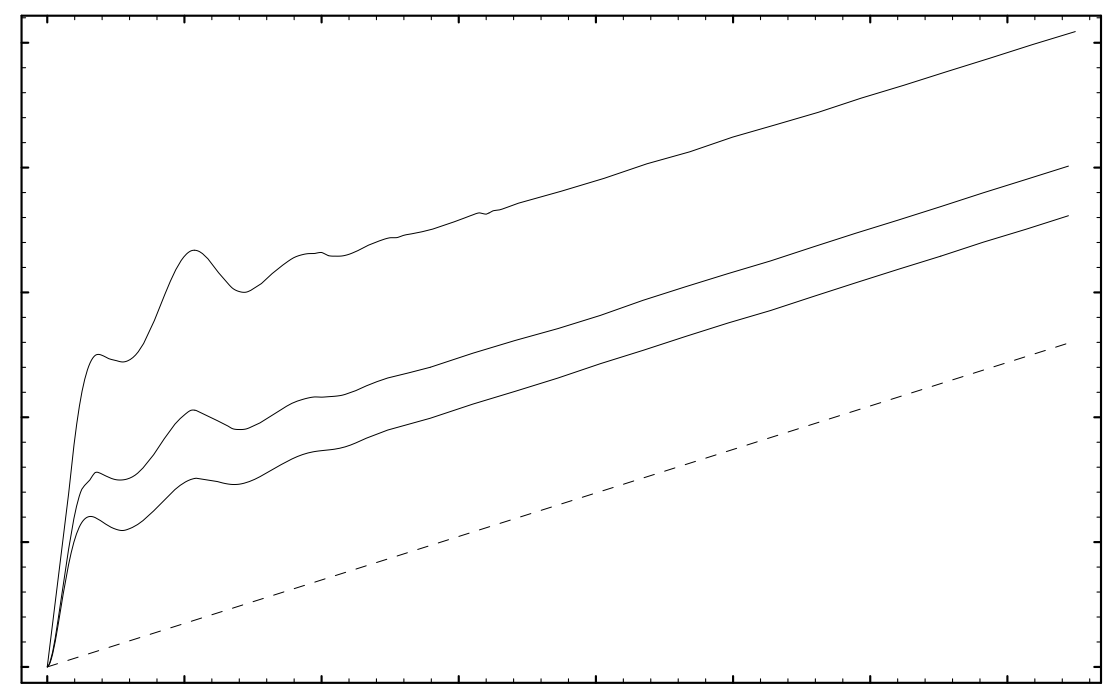

Figure 2: The average number of created bosons for $\bar{m}=1$. The dashed line shows the asymptotic behaviour at zero temperature. All quantities are expressed in units of the electric field.

$n\left(\mathbf{p}, t_{f}\right)$ as

$$
n=\frac{1}{2 \bar{\omega}_{0} \bar{\omega}_{0}^{g}}\left(\left(\bar{\omega}_{0} r_{g}-\frac{\bar{\omega}_{0}^{g}}{r_{g}}\right)^{2}+\dot{r}_{g}^{2}\right)
$$

Equations (79) and (80) constitute the exact expressions for the expectation value of the numbers of particles created by the external field at finite temperature, when a time $t_{f}$ has elapsed. Since, as we have argued, the positively charged particles created by $\hat{b}^{\dagger}(\mathbf{p})$ have a momentum $(-\mathbf{p})$, their momentum distribution at zero temperature will be given by $n(-\mathbf{p}) / 2=\left\langle\hat{b}^{\dagger}(-\mathbf{p}) \hat{b}(-\mathbf{p})\right\rangle_{\beta=\infty}$. Figure 1 shows this distribution. Note that for small times, there are very few particles with zero momentum. The result of integrating (79) numerically for a mass $\bar{m}=1$ is shown in figure 2. We see that increasing the temperature increases the number of created particles but for large enough $t_{f}$ the slope is independent of temperature.

We have so far only considered a simple time dependence of the electromagnetic field, but it is straightforward to generalize to an arbitrary time dependence. Introduce the two dimensionless functions $f(\bar{t}), F(\bar{t})$ and let

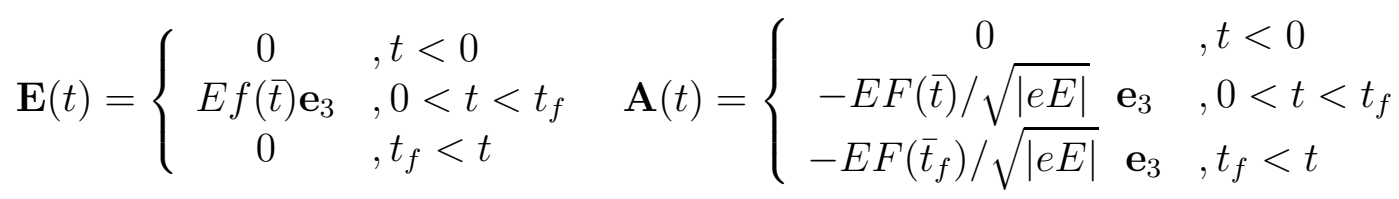

The functions $f(\bar{t})$ and $F(\bar{t})$ must satisfy

$$
\dot{F}(\bar{t})=f(\bar{t}) \quad, \quad F(0)=0
$$


As a matter of fact, (79) and (80) still hold, with the modifications that $r_{g}$ satisfies the gauge transformed version of (57), where now

$$
\bar{\omega}^{g}=\sqrt{\Lambda+\left(\bar{p}^{3}-F(\bar{t})+F\left(\bar{t}_{f}\right)\right)^{2}}
$$

and similarily for $\bar{\omega}_{0}$ and $\bar{\omega}_{0}^{g}$. Because of the new time dependence, however, $r_{g}$ cannot be expressed in terms of special functions, but has to be found numerically. This is in practice not a serious drawback, since the integral still must be evaluated numerically.

\subsection{Asymptotic expansions}

We have obtained the exact expression for the number of particles created for arbitrary times $t_{f}$. It is, however, possible to obtain some more convenient expressions when $t_{f}$ is large. To that end we investigate the behaviour of the number density $n$. The number density may be written

$$
n\left(\Lambda, \bar{p}^{3}, \bar{t}_{f}\right)=\frac{\bar{\omega}_{0}^{2}\left|z_{g}\left(\bar{t}_{f}\right)\right|^{2}+\left|\dot{z}_{g}\left(\bar{t}_{f}\right)\right|^{2}}{i \bar{\omega}_{0}\left(\dot{z}_{g}^{*}\left(\bar{t}_{f}\right) z_{g}\left(\bar{t}_{f}\right)-\dot{z}_{g}\left(\bar{t}_{f}\right) z_{g}^{*}\left(\bar{t}_{f}\right)\right)}-1
$$

where $z_{g}(\bar{t})$ by (59) satisfies

$$
\ddot{z}_{g}+\left(\bar{t}-\bar{t}_{f}-\bar{p}^{3}\right)^{2} z_{g}=0 .
$$

Since the number density $n$ is not sensitive to a rescaling of $z_{g}$, the relevant initial condition for $z_{g}$ following from (59) is

$$
\frac{\dot{z}_{g}(0)}{z_{g}(0)}=i \bar{\omega}_{0}^{g}
$$

Let us now be more specific and consider a large final time $\bar{t}_{f}>>1$. For such $\bar{t}_{f}$, consider $\Lambda$ and $\bar{p}^{3}$ satisfying

$$
-\bar{t}_{f}-\sqrt{\Lambda}<<\bar{p}^{3}<<-\sqrt{\Lambda}, \quad \bar{p}^{3}<<-1, \quad \bar{t}_{f}>>\sqrt{\Lambda} .
$$

For $\Lambda$ and $\bar{p}^{3}$ restricted by (87) the initial condition (86) reads

$$
\frac{\dot{z}_{g}(0)}{z_{g}(0)} \sim i\left(\bar{t}_{f}+\bar{p}^{3}\right)
$$

A solution to (85) satisfying (88) is

$$
z_{g}(\bar{t})=D_{-\frac{i}{2} \Lambda-\frac{1}{2}}\left(-(1+i)\left(\bar{t}-\bar{t}_{f}-\bar{p}^{3}\right)\right)
$$

which may be confirmed using the first of the following asymptotic expansions of parabolic cylinder functions when $|x|>>1,|x|>>|\nu|[13]$ :

$$
\begin{aligned}
& D_{\nu}\left(x e^{\frac{i \pi}{4}}\right) \sim e^{-\frac{i x^{2}}{4}}\left(x e^{\frac{i \pi}{4}}\right)^{\nu} \quad, \quad x>0 \\
& D_{\nu}\left(x e^{\frac{i \pi}{4}}\right) \sim e^{-\frac{i x^{2}}{4}}\left(x e^{\frac{i \pi}{4}}\right)^{\nu}-\frac{\sqrt{2 \pi}}{\Gamma(-\nu)} e^{\frac{i x^{2}}{4}} e^{-i \pi \nu}\left(x e^{\frac{i \pi}{4}}\right)^{-\nu-1}, \quad x<0 .
\end{aligned}
$$


It is now a straightforward calculation to find the asymptotic behaviour of $z_{g}\left(\bar{t}_{f}\right)$ in which case (91) applies. By (84), and using $\bar{\omega}_{0} \sim\left|\bar{p}^{3}\right|$ and $\left|\Gamma\left(\frac{i}{2} \Lambda+\frac{1}{2}\right)\right|^{-2}=\frac{1}{\pi} \cosh \frac{\pi \Lambda}{2}$, one finds the number density

$$
n\left(\Lambda, \bar{p}^{3}, \bar{t}_{f}\right) \sim 2 e^{-\pi \Lambda} .
$$

What happens if $\bar{p}$ lies outside the region in (87)? It is clear that for $\left|\bar{p}^{3}\right|$ sufficiently large compared to $\bar{t}_{f}$, we can neglect the time dependence in the equation for $z_{g}$. Hence there will be no created particles i.e. $n\left(\Lambda, \bar{p}^{3}, \bar{t}_{f}\right)=0$. In the region where $|\mathbf{p}|$ is neither large, nor restricted by (87), the contribution to $\langle\hat{N}\rangle^{\mathrm{cr}}$ will be small compared to the dominant contribution. Therefore we can effectively set $n$ to zero in this region. We conclude that for $\bar{t}_{f}>>1$ and $\bar{t}_{f}>>\bar{m}$,

$$
n\left(\Lambda, \bar{p}^{3}, \bar{t}_{f}\right)= \begin{cases}2 e^{-\pi \Lambda}, & -\bar{t}_{f}<\bar{p}^{3}<0 \\ 0, & \text { otherwise }\end{cases}
$$

and thus

$$
\langle\hat{N}\rangle^{\mathrm{cr}}\left(\bar{t}_{f}\right)=\frac{\bar{V}}{2(2 \pi)^{2}} \int_{-t_{f}}^{0} d \bar{p}^{3} \int_{\bar{m}^{2}}^{\infty} d \Lambda 2 e^{-\pi \Lambda} \operatorname{coth} \frac{\bar{\beta} \bar{\omega}_{0}^{g}}{2} .
$$

Two limits involving $\beta$ may now be considered, namely $1 / \bar{\beta}<<\bar{t}_{f}$ and $1 / \bar{\beta}>>\bar{t}_{f}$,

$$
\begin{aligned}
\langle\hat{N}\rangle^{\mathrm{cr}}\left(\bar{t}_{f}\right) & =\frac{\bar{V}}{4 \pi^{3}} \bar{t}_{f} e^{-\pi \bar{m}^{2}}=\frac{\alpha}{\pi^{2}} V E^{2} t_{f} e^{-\frac{\pi m^{2}}{e E \mid}}, \frac{1}{\bar{\beta}}<<\bar{t}_{f} \\
\langle\hat{N}\rangle^{\mathrm{cr}}\left(\bar{t}_{f}\right) & =\frac{\bar{V}}{2 \bar{\beta} \pi^{3}} \ln \left(2 \bar{t}_{f}\right) e^{-\pi \bar{m}^{2}}=\left(\frac{\alpha}{\pi}\right)^{1 / 2} \frac{V|E|}{\beta \pi^{2}} \ln \left(2 t_{f} \sqrt{|e E|}\right) e^{-\frac{\pi m^{2}}{e E \mid}}, \quad \frac{1}{\bar{\beta}}>>\bar{t}_{f}(96)
\end{aligned}
$$

The result (95) is identical to the zero temperature result obtained in [14], eq. (5.1.61).

\subsection{Probabilities}

The probability $P(\Psi)$ of finding the system in a normalized state $|\Psi\rangle$ is

$$
P(\Psi)=\langle\Psi|\hat{\rho}| \Psi\rangle
$$

We are interested in the probability $P\left(n, m ; t_{f}\right)$ of finding the system in a state with $n$ particles and $m$ anti particles at time $t=t_{f}$. For specific momenta these states are

$$
\left|\mathbf{p}_{1} \ldots \mathbf{p}_{n}, \mathbf{k}_{1} \ldots \mathbf{k}_{m}\right\rangle=\frac{1}{V^{(n+m) / 2}} \frac{1}{\sqrt{n ! m !}} \prod_{i=1}^{n} \prod_{j=1}^{m} \hat{a}^{\dagger}\left(\mathbf{p}_{i}\right) \hat{b}^{\dagger}\left(\mathbf{k}_{j}\right)|0\rangle .
$$

The desired probability is a sum of probabilities for such states with $n$ and $m$ held fixed, i.e.

$$
P\left(n, m ; t_{f}\right)=V^{n+m} \int_{p_{1} \ldots p_{n}} \int_{k_{1} \ldots k_{m}}\left\langle\mathbf{p}_{1} \ldots \mathbf{p}_{n}, \mathbf{k}_{1} \ldots \mathbf{k}_{m}\left|\hat{\rho}\left(t_{f}\right)\right| \mathbf{p}_{1} \ldots \mathbf{p}_{n}, \mathbf{k}_{1} \ldots \mathbf{k}_{m}\right\rangle
$$

One finds that the probabilities, $P\left(n, m ; t_{f}\right)$, are generated by a function $f\left(x, y ; t_{f}\right)$ as

$$
P\left(n, m ; t_{f}\right)=\left.f\left(1,1 ; t_{f}\right)^{-1} \frac{1}{n ! m !} \frac{\partial^{n+m}}{\partial x^{n} \partial y^{m}} f\left(x, y ; t_{f}\right)\right|_{x=y=0}
$$


It immediately follows that $\sum_{n, m=0}^{\infty} P\left(n, m ; t_{f}\right)=1$. The generating function $f\left(x, y ; t_{f}\right)$ reads

$$
f\left(x, y ; t_{f}\right)=\exp \left[-\operatorname{Tr} \ln \left\{\left(1-x g\left(t_{f}\right)\right)\left(1-y g\left(t_{f}\right)\right)-x y h\left(t_{f}\right)\right\}\right]
$$

where the functions $g\left(t_{f}\right)$ and $h\left(t_{f}\right)$ are expressed in terms of the number density $n\left(t_{f}\right)$ defined in (80). Suppressing the momentum dependence, we have

$$
\begin{aligned}
g\left(t_{f}\right) & =\frac{1}{\left(n\left(t_{f}\right)+1+\operatorname{coth} \bar{\beta} \bar{\omega}_{0}^{g}\right) \sinh \bar{\beta} \bar{\omega}_{0}^{g}} \\
h\left(t_{f}\right) & =\frac{n\left(t_{f}\right)\left(n\left(t_{f}\right)+2\right)}{\left(n\left(t_{f}\right)+1+\operatorname{coth} \bar{\beta} \bar{\omega}_{0}^{g}\right)^{2}}
\end{aligned}
$$

We note that when $\bar{\beta}=\infty$ only $h\left(t_{f}\right)$ survives. Since $h\left(t_{f}\right)$ multiplies $x y$ we conclude that only uncharged states are present at zero temperature. This is what we expect since all states have been created out of the uncharged groundstate. On the other hand when $t_{f}=0$ we have that $h(0)=0$ while $g(0)=\exp \left(-\beta \omega_{0}\right)$, leading to ordinary Boltzmann probabilities for the initial ensemble.

The probability $P\left(0,0 ; t_{f}\right)$ is readily obtained

$$
\begin{aligned}
P\left(0,0 ; t_{f}\right) & =f\left(1,1 ; t_{f}\right)^{-1} \\
& =\exp \left[-\operatorname{Tr} \ln \left\{\frac{1}{2} \operatorname{coth}\left(\frac{\bar{\beta} \bar{\omega}_{0}^{g}}{2}\right)\left(n\left(t_{f}\right)+1+\operatorname{coth} \bar{\beta} \bar{\omega}_{0}^{g}\right)\right\}\right]
\end{aligned}
$$

At zero temperature this reduces to

$$
P\left(0,0 ; t_{f}\right)=\exp \left[-\operatorname{Tr} \ln \left(1+\frac{n\left(t_{f}\right)}{2}\right)\right], \bar{\beta}=\infty
$$

which for large times, $\bar{t}_{f}>>1$, by (93) becomes

$$
P\left(0,0 ; t_{f}\right)=\exp \left[-\frac{\bar{V} \bar{t}_{f}}{2(2 \pi)^{2}} \int_{\bar{m}^{2}}^{\infty} d \Lambda \ln \left(1+e^{-\pi \Lambda}\right)\right]
$$

This is the result obtained by Schwinger in 1951 [1]. We end this section by noting that expectation values of some operators may be obtained from the generating function. For the number operator we have

$$
\begin{aligned}
\langle\hat{N}\rangle\left(t_{f}\right) & =\sum_{n, m=0}^{\infty}(n+m) P\left(n, m ; t_{f}\right) \\
& =\left.f\left(1,1 ; t_{f}\right)^{-1} \partial_{x} f\left(x, x ; t_{f}\right)\right|_{x=1} \\
& =\operatorname{Tr}\left[\left(n\left(t_{f}\right)+1\right) \operatorname{coth} \frac{\bar{\beta} \bar{\omega}_{0}^{g}}{2}-1\right]
\end{aligned}
$$

which of course agrees with (77). 


\section{$3 \quad$ Fermions}

We will set up the formalism by studying an illustrative example, the two-dimensional fermionic harmonic oscillator. The annihilation and creation operators, $\hat{a}, \hat{b}$ and $\hat{a}^{\dagger}, \hat{b}^{\dagger}$, satisfy

$$
\left\{\hat{a}, \hat{a}^{\dagger}\right\}=\left\{\hat{b}, \hat{b}^{\dagger}\right\}=1 \text {. }
$$

All other anticommutators vanish. The Hilbert space is spanned by the four orthogonal states $|0,0\rangle,|1,0\rangle,|0,1\rangle$ and $|1,1\rangle$,

$$
\hat{a}|0,0\rangle=\hat{b}|0,0\rangle=0, \quad \hat{a}^{\dagger}|0,0\rangle=|1,0\rangle, \quad \hat{b}^{\dagger}|0,0\rangle=|0,1\rangle, \quad \hat{a}^{\dagger} \hat{b}^{\dagger}|0,0\rangle=|1,1\rangle .
$$

The states are normalized, $\langle 0,0 \mid 0,0\rangle=\langle 1,0 \mid 1,0\rangle=\langle 0,1 \mid 0,1\rangle=\langle 1,1 \mid 1,1\rangle=1$. We represent this system by functions $\Psi\left(\eta^{*}, \eta\right)$ of a complex Grassmann number $\eta$ and its complex conjugate $\eta^{*}$ in the following manner,

$$
\begin{aligned}
& |0,0\rangle \leftrightarrow 1, \quad|1,0\rangle \leftrightarrow \eta^{*}, \quad|0,1\rangle \leftrightarrow \eta, \quad|1,1\rangle \leftrightarrow \eta^{*} \eta, \\
& \hat{a} \leftrightarrow \partial_{\eta^{*}}, \quad \hat{a}^{\dagger} \leftrightarrow \eta^{*}, \quad \hat{b} \leftrightarrow \partial_{\eta}, \quad \hat{b}^{\dagger} \leftrightarrow \eta,
\end{aligned}
$$

so that overlaps with Grassmann states are given by

$$
\left\langle\eta^{*} \eta \mid 0,0\right\rangle=1, \quad\left\langle\eta^{*} \eta \mid 1,0\right\rangle=\eta^{*}, \quad\left\langle\eta^{*} \eta \mid 0,1\right\rangle=\eta, \quad\left\langle\eta^{*} \eta \mid 1,1\right\rangle=\eta^{*} \eta
$$

and

$$
\begin{aligned}
\left\langle\eta^{*}, \eta \mid \eta^{\prime *}, \eta^{\prime}\right\rangle & =\sum_{n, m=0}^{1}\left\langle\eta^{*}, \eta \mid n, m\right\rangle\left\langle n, m \mid \eta^{*}, \eta^{\prime}\right\rangle \\
& =1+\eta^{*} \eta^{\prime}-\eta^{\prime *} \eta+\eta^{*} \eta \eta^{\prime *} \eta^{\prime}=e^{\eta^{*} \eta^{\prime}-\eta^{\prime *} \eta}
\end{aligned}
$$

The Grassmann state may now be expanded as

$$
\left|\eta^{*} \eta\right\rangle=\sum_{n, m=0}^{1}|n, m\rangle\left\langle n, m \mid \eta^{*} \eta\right\rangle=|0,0\rangle+|1,0\rangle \eta+|0,1\rangle \eta^{*}+|1,1\rangle \eta^{*} \eta .
$$

The partition of unity can thus be expressed as

$$
\begin{aligned}
\hat{1} & =|0,0\rangle\langle 0,0|+| 1,0\rangle\langle 1,0|+| 0,1\rangle\langle 0,1|+| 1,1\rangle\langle 1,1| \\
& =\int d^{2} \eta^{\prime} d^{2} \eta\left|\eta^{*} \eta\right\rangle\left\langle\eta^{*} \eta \mid \eta^{\prime *} \eta^{\prime}\right\rangle\left\langle\eta^{\prime *} \eta^{\prime}\right|
\end{aligned}
$$

where $d^{2} \eta=d \eta^{*} d \eta$. Hence an arbitrary overlap is given by

$$
\begin{aligned}
\left\langle\Psi_{1} \mid \Psi_{2}\right\rangle & =\int d^{2} \eta^{\prime} d^{2} \eta\left\langle\Psi_{1} \mid \eta^{*} \eta\right\rangle\left\langle\eta^{*} \eta \mid \eta^{\prime *} \eta^{\prime}\right\rangle\left\langle\eta^{\prime *} \eta^{\prime} \mid \Psi_{2}\right\rangle \\
& =\int d^{2} \eta^{\prime} d^{2} \eta e^{\eta^{*} \eta^{\prime}-\eta^{\prime *} \eta} \Psi_{1}^{*}\left(\eta^{*}, \eta\right) \Psi_{2}\left(\eta^{\prime *}, \eta^{\prime}\right) .
\end{aligned}
$$


Let $\hat{\mathcal{O}}$ be an operator,

$$
\hat{\mathcal{O}}=\sum_{n_{1}, n_{2}, m_{1}, m_{2}=0}^{1} \mathcal{O}_{n_{1} n_{2}, m_{1} m_{2}}\left|n_{1}, n_{2}\right\rangle\left\langle m_{1}, m_{2}\right| .
$$

The trace of this operator is then,

$$
\operatorname{tr} \hat{\mathcal{O}}=\mathcal{O}_{00,00}+\mathcal{O}_{10,10}+\mathcal{O}_{01,01}+\mathcal{O}_{11,11}
$$

This can be expressed as,

$$
\operatorname{tr} \hat{\mathcal{O}}=\int d^{2} \eta^{\prime} d^{2} \eta\left\langle\eta^{*} \eta|\hat{\mathcal{O}}| \eta^{\prime *} \eta^{\prime}\right\rangle\left\langle\eta^{*} \eta \mid \eta^{\prime *} \eta^{\prime}\right\rangle
$$

The "naive" trace,

$$
\int d^{2} \eta^{\prime} d^{2} \eta\left\langle\eta^{\prime *} \eta^{\prime}|\hat{\mathcal{O}}| \eta^{*} \eta\right\rangle\left\langle\eta^{*} \eta \mid \eta^{\prime *} \eta^{\prime}\right\rangle=\mathcal{O}_{00,00}-\mathcal{O}_{10,10}-\mathcal{O}_{01,01}+\mathcal{O}_{11,11}
$$

is in fact the supertrace $\operatorname{Str}(\hat{\mathcal{O}})=\operatorname{tr}\left(\hat{\mathcal{O}}(-1)^{\hat{F}}\right)$. Let us now turn to field theory. The Hamiltonian for Dirac fermions in an external electromagnetic field $\mathbf{A}$ is,

$$
\left.\hat{H}=\int d^{3} x d^{3} y \frac{1}{2} \hat{[} \psi_{\alpha}^{\dagger}(\mathbf{x}), \hat{\psi}_{\beta}(\mathbf{y})\right] h_{\alpha \beta}(\mathbf{x}, \mathbf{y})
$$

where

$$
h(\mathbf{x}, \mathbf{y})=\left(-i \gamma^{0} \boldsymbol{\gamma} \cdot\left(\nabla_{x}-i e \mathbf{A}(\mathbf{x})\right)+m \gamma^{0}\right) \delta(\mathbf{x}-\mathbf{y})
$$

The anticommutation relations read

$$
\left\{\hat{\psi}_{\alpha}(\mathbf{x}), \hat{\psi}_{\beta}^{\dagger}(\mathbf{y})\right\}=\delta_{\alpha \beta} \delta(\mathbf{x}-\mathbf{y}) .
$$

All other anticommutators vanish. As was first done in [9], we let the fermionic field operators act on wavefunctionals $\Psi\left(\eta^{*}, \eta\right)$ of a complex Grassmann field $\eta$ and its complex conjugate $\eta^{*}$ according to

$$
\begin{aligned}
\hat{\psi}_{\alpha}(\mathbf{x}) & \leftrightarrow \frac{1}{\sqrt{2}}\left(\eta_{\alpha}(\mathbf{x})+\frac{\delta}{\delta \eta_{\alpha}^{*}(\mathbf{x})}\right) \\
\hat{\psi}_{\alpha}^{\dagger}(\mathbf{x}) & \leftrightarrow \frac{1}{\sqrt{2}}\left(\eta_{\alpha}^{*}(\mathbf{x})+\frac{\delta}{\delta \eta_{\alpha}(\mathbf{x})}\right)
\end{aligned}
$$

The representation defined by (124) and (125) of the field operator algebra is reducible. This can be seen explicitly by introducing the operators,

$$
\begin{aligned}
& \hat{\theta}_{\alpha}(\mathbf{x}) \leftrightarrow \frac{1}{\sqrt{2}}\left(\eta_{\alpha}(\mathbf{x})-\frac{\delta}{\delta \eta_{\alpha}^{*}(\mathbf{x})}\right) \\
& \hat{\theta}_{\alpha}^{\dagger}(\mathbf{x}) \leftrightarrow \frac{1}{\sqrt{2}}\left(-\eta_{\alpha}^{*}(\mathbf{x})+\frac{\delta}{\delta \eta_{\alpha}(\mathbf{x})}\right)
\end{aligned}
$$


We have

$$
\left\{\hat{\theta}_{\alpha}(\mathbf{x}), \hat{\theta}_{\beta}^{\dagger}(\mathbf{y})\right\}=\delta_{\alpha \beta} \delta(\mathbf{x}-\mathbf{y})
$$

and $\hat{\theta}$ as well as $\hat{\theta}^{\dagger}$ anticommute with both $\hat{\psi}$ and $\hat{\psi}^{\dagger}$ making the representation reducible. Contrary to what is said in [9] we may now view wavefunctionals as overlaps with Grassmann field states $\left|\eta^{*} \eta\right\rangle$,

$$
\begin{aligned}
\left\langle\eta^{*} \eta \mid \eta^{\prime *} \eta^{\prime}\right\rangle & =\exp \left(\int d^{3} x\left(\eta_{\alpha}^{*}(\mathbf{x}) \eta_{\alpha}^{\prime}(\mathbf{x})-\eta_{\alpha}^{\prime *}(\mathbf{x}) \eta_{\alpha}(\mathbf{x})\right)\right) \\
\Psi\left(\eta^{*}, \eta\right) & =\left\langle\eta^{*} \eta \mid \Psi\right\rangle .
\end{aligned}
$$

The partition of unity is given by functional Grassmann integration,

$$
\hat{1}=\int D^{2} \eta^{\prime} D^{2} \eta\left|\eta^{*} \eta\right\rangle\left\langle\eta^{*} \eta \mid \eta^{*} \eta^{\prime}\right\rangle\left\langle\eta^{\prime *} \eta^{\prime}\right|,
$$

having set $D^{2} \eta=D \eta^{*} D \eta$ and hence the inner product is

$$
\left\langle\Psi_{1} \mid \Psi_{2}\right\rangle=\int D^{2} \eta^{\prime} D^{2} \eta\left\langle\eta^{*} \eta \mid \eta^{\prime *} \eta^{\prime}\right\rangle \Psi_{1}^{*}\left(\eta^{*}, \eta\right) \Psi_{2}\left(\eta^{\prime *}, \eta^{\prime}\right) .
$$

For an operator $\hat{\mathcal{O}}$ we have

$$
\begin{aligned}
\left\langle\eta^{*} \eta|\hat{\mathcal{O}}| \eta^{\prime *} \eta^{\prime}\right\rangle & =\mathcal{O}\left(\eta^{*} \eta, \eta^{\prime *} \eta^{\prime}\right) \\
\left\langle\eta^{*} \eta\left|\hat{\mathcal{O}}\left(\hat{\psi}, \hat{\psi}^{\dagger}\right)\right| \Psi\right\rangle & =\hat{\mathcal{O}}\left(\eta^{*}, \frac{\delta}{\delta \eta^{*}}, \eta, \frac{\delta}{\delta \eta}\right)\left\langle\eta^{*} \eta \mid \Psi\right\rangle, \\
\operatorname{tr}_{d}(\hat{\mathcal{O}}) & =\int D^{2} \eta^{\prime} D^{2} \eta\left\langle\eta^{*} \eta|\hat{\mathcal{O}}| \eta^{\prime *} \eta^{\prime}\right\rangle\left\langle\eta^{*} \eta \mid \eta^{\prime *} \eta^{\prime}\right\rangle
\end{aligned}
$$

Since the representation is reducible, all states will have an unphysical degeneracy with the degree of degeneracy given by $d$. Hence the physical trace is given by

$$
\operatorname{tr}(\hat{\mathcal{O}})=\frac{1}{d} \operatorname{tr}_{d}(\hat{\mathcal{O}})
$$

The degeneracy $d$ is given by the number of states generated by $\hat{\theta}^{\dagger}$ and $\hat{\theta}$. For symmetry reasons this is equal to the number of states generated by $\hat{\psi}^{\dagger}$ and $\hat{\psi}$. The total number of states in the functional space is $\operatorname{tr}_{d}(\hat{1})$. Hence,

$$
d^{2}=\operatorname{tr}_{d}(\hat{1})=\int D^{2} \eta^{\prime} D^{2} \eta\left\langle\eta^{*} \eta \mid \eta^{*} \eta^{\prime}\right\rangle^{2}=\operatorname{det}(4 I)=\exp \left(4 V \int_{p} \ln 4\right),
$$

where $I_{\alpha \beta}(\mathbf{x}, \mathbf{y})=\delta_{\alpha \beta} \delta(\mathbf{x}-\mathbf{y})$. Thus

$$
d=\operatorname{det}(2 I)=\exp \left(4 V \int_{p} \ln 2\right) .
$$

Let us end this subsection by giving a standard formula for Gaussian functional integrals,

$$
\begin{gathered}
\int D^{2} \eta \exp \left(\int d^{3} x d^{3} y \eta_{\alpha}^{*}(\mathbf{x}) \Omega_{\alpha \beta}(\mathbf{x}, \mathbf{y}) \eta_{\beta}(\mathbf{y})+\int d^{3} x \eta_{\alpha}^{*}(\mathbf{x}) \chi_{\alpha}(\mathbf{x})+\xi_{\alpha}^{*}(\mathbf{x}) \eta_{\alpha}(\mathbf{x})\right)= \\
\operatorname{det}(-\Omega) \exp \left(-\int d^{3} x d^{3} y \xi_{\alpha}^{*}(\mathbf{x}) \Omega_{\alpha \beta}^{-1}(\mathbf{x}, \mathbf{y}) \chi_{\beta}(\mathbf{y})\right)
\end{gathered}
$$




\subsection{Initial ensemble}

We will now do all the calculations for fermions that were previously done for bosons. Let $\hat{H}_{0}$ denote the Hamiltonian with $\mathbf{A}=0$. By (16) and (17) we find, letting $\rho_{u}\left(\eta_{1}^{*} \eta_{1}, \eta_{2}^{*} \eta_{2}\right)=\left\langle\eta_{1}^{*}, \eta_{1}\left|\hat{\rho}_{u}\right| \eta_{2}^{*}, \eta_{2}\right\rangle$

$$
\begin{aligned}
-\frac{\partial}{\partial \beta} \rho_{u}\left(\eta_{1}^{*} \eta_{1}, \eta_{2}^{*} \eta_{2}\right) & =\hat{H}_{0}\left(\eta_{1}^{*}, \frac{\delta}{\delta \eta_{1}^{*}}, \eta_{1}, \frac{\delta}{\delta \eta_{1}}\right) \rho_{u}\left(\eta_{1}^{*} \eta_{1}, \eta_{2}^{*} \eta_{2}\right) \\
\lim _{\beta \rightarrow 0} \rho_{u}\left(\eta_{1}^{*} \eta_{1}, \eta_{2}^{*} \eta_{2}\right) & =\left\langle\eta_{1}^{*}, \eta_{1} \mid \eta_{2}^{*}, \eta_{2}\right\rangle \\
\rho_{u}\left(\eta_{1}^{*} \eta_{1}, \eta_{2}^{*} \eta_{2}\right) & =\rho_{u}^{*}\left(\eta_{2}^{*} \eta_{2}, \eta_{1}^{*} \eta_{1}\right) .
\end{aligned}
$$

Make a Gaussian ansatz (suppressing spinor indices),

$$
\rho_{u}\left(\eta_{1}^{*} \eta_{1}, \eta_{2}^{*} \eta_{2}\right)=N_{u} \exp \left(\int d^{3} x d^{3} y \eta_{i}^{*}(\mathbf{x}) \Omega_{i j}^{0}(\mathbf{x}, \mathbf{y}) \eta_{j}(\mathbf{y})\right) \quad i, j=1,2 .
$$

Using (136) we obtain

$$
\begin{aligned}
-\partial_{\beta} \ln N_{u} & =\frac{1}{2} \operatorname{Tr}\left(h^{0} \Omega_{11}^{0}\right), \\
-\partial_{\beta} \Omega_{11}^{0} & =\frac{1}{2}\left(I-\Omega_{11}^{0}\right) h^{0}\left(I+\Omega_{11}^{0}\right), \\
-\partial_{\beta} \Omega_{12}^{0} & =\frac{1}{2}\left(I-\Omega_{11}^{0}\right) h^{0} \Omega_{12}^{0} \\
-\partial_{\beta} \Omega_{21}^{0} & =-\frac{1}{2} \Omega_{21}^{0} h^{0}\left(I+\Omega_{11}^{0}\right), \\
-\partial_{\beta} \Omega_{22}^{0} & =-\frac{1}{2} \Omega_{21}^{0} h^{0} \Omega_{12}^{0}
\end{aligned}
$$

where $h^{0}$ is $h$ having set $\mathbf{A}=0$. Furthermore, by (137),

$$
\begin{aligned}
& \lim _{\beta \rightarrow 0} N_{u}=1, \\
& \lim _{\beta \rightarrow 0} \Omega_{11}^{0}=\lim _{\beta \rightarrow 0} \Omega_{22}^{0}=0, \\
& \lim _{\beta \rightarrow 0} \Omega_{12}^{0}=-\lim _{\beta \rightarrow 0} \Omega_{21}^{0}=I,
\end{aligned}
$$

and by (138),

$$
\left(\Omega_{11}^{0}\right)^{\dagger}=\Omega_{22}^{0}, \quad\left(\Omega_{12}^{0}\right)^{\dagger}=\Omega_{12}^{0}, \quad\left(\Omega_{21}^{0}\right)^{\dagger}=\Omega_{21}^{0},
$$

where $\left(\Omega^{\dagger}\right)_{\alpha \beta}(\mathbf{x}, \mathbf{y})=\Omega_{\beta \alpha}^{*}(\mathbf{y}, \mathbf{x})$. Fourier transforming, just like for bosons,

$$
\Omega(\mathbf{x}, \mathbf{y})=\int_{p} \Omega(\mathbf{p}) e^{i \mathbf{p} \cdot(\mathbf{x}-\mathbf{y})}, \quad \eta(\mathbf{x})=\int_{p} \eta(\mathbf{p}) e^{i \mathbf{p} \cdot \mathbf{x}},
$$

we find the solution satisfying (140)-(148),

$$
\begin{aligned}
\Omega_{11}^{0}(\mathbf{p}) & =\Omega_{22}^{0}(\mathbf{p})=-\gamma^{0} \frac{(\boldsymbol{\gamma} \cdot \mathbf{p}+m)}{\omega_{0}} \tanh \left(\frac{\beta \omega_{0}}{2}\right), \\
\Omega_{12}^{0}(\mathbf{p}) & =\Omega_{11}(\mathbf{p})+1, \quad \Omega_{21}^{0}(\mathbf{p})=\Omega_{11}^{0}(\mathbf{p})-1 \\
N_{u} & =\exp \left[4 V \int_{p} \ln \cosh \frac{\beta \omega_{0}}{2}\right] .
\end{aligned}
$$


Hence one may obtain the partition function,

$$
Z=\operatorname{tr} \hat{\rho}_{u}=\exp \left[4 V \int_{p} \ln 2 \cosh \frac{\beta \omega_{0}}{2}\right],
$$

and the normalized density matrix, $\hat{\rho}_{0}=\hat{\rho}_{u} / \operatorname{tr} \hat{\rho}_{u}$,

$$
\begin{aligned}
& \rho_{0}\left(\eta_{1}^{*} \eta_{1}, \eta_{2}^{*} \eta_{2}\right)=\exp \left(-4 V \int_{p} \ln 2\right) \times \\
& \quad \exp \left[\int_{p}\left(\left(\eta_{1}^{*}+\eta_{2}^{*}\right) \Omega_{11}^{0}\left(\eta_{1}+\eta_{2}\right)+\eta_{1}^{*} \eta_{2}-\eta_{2}^{*} \eta_{1}\right)\right],
\end{aligned}
$$

having suppressed the momentum dependence.

\subsection{The time dependent ensemble}

Let us now find $\hat{\rho}(t)$ with the initial condition $\hat{\rho}(0)=\hat{\rho}_{0}$. By (40), one has

$$
i \partial_{t} \rho\left(\eta_{1}^{*} \eta_{1}, \eta_{2}^{*} \eta_{2}\right)=\hat{H}\left(\eta_{1}^{*}, \frac{\delta}{\delta \eta_{1}^{*}}, \eta_{1}, \frac{\delta}{\delta \eta_{1}}\right) \rho\left(\eta_{1}^{*} \eta_{1}, \eta_{2}^{*} \eta_{2}\right)-\left(\hat{H}\left(\eta_{2}^{*}, \frac{\delta}{\delta \eta_{2}^{*}}, \eta_{2}, \frac{\delta}{\delta \eta_{2}}\right) \rho\left(\eta_{2}^{*} \eta_{2}, \eta_{1}^{*} \eta_{1}\right)\right)^{*}
$$

We make an ansatz of the same form as the initial condition,

$$
\begin{aligned}
& \rho\left(\eta_{1}^{*} \eta_{1}, \eta_{2}^{*} \eta_{2}, t\right)=\exp \left(-4 V \int_{p} \ln 2\right) \times \\
& \quad \exp \left[\int_{p}\left(\left(\eta_{1}^{*}+\eta_{2}^{*}\right) \tanh \left(\frac{\beta \omega_{0}}{2}\right) \Omega(t)\left(\eta_{1}+\eta_{2}\right)+\eta_{1}^{*} \eta_{2}-\eta_{2}^{*} \eta_{1}\right)\right],
\end{aligned}
$$

in momentum space. Since $\hat{\rho}$ is hermitian, $\Omega(\mathbf{p}, t)$ is a hermitian matrix. Hence, with the same external field as for bosons, using (150) we obtain

$$
i \partial_{t} \Omega=[h, \Omega], \quad \Omega(\mathbf{p}, 0)=-\gamma^{0} \frac{(\boldsymbol{\gamma} \cdot \mathbf{p}+m)}{\omega_{0}(\mathbf{p})} .
$$

Introducing the spinors $\chi_{1}$ and $\chi_{2}$,

$$
1+\Omega=\chi_{1} \chi_{1}^{\dagger}+\chi_{2} \chi_{2}^{\dagger}
$$

where,

$$
i \partial_{t} \chi_{j}=h \chi_{j}, \quad j=1,2
$$

we solve (157). Noting that,

$$
h(\mathbf{p})=\gamma^{0}(\boldsymbol{\gamma} \cdot(\mathbf{p}-e \mathbf{A})+m)
$$

and hence $h(\mathbf{p})^{2}=\omega(\mathbf{p})^{2}=(\mathbf{p}-e \mathbf{A})^{2}+m^{2}$, we differentiate (159) with respect to $\mathrm{t}$ and obtain,

$$
\frac{\partial^{2} \chi_{j}}{\partial t^{2}}+\left(\omega^{2}+i e E \gamma^{0} \gamma^{3}\right) \chi_{j}=0
$$


To have (160) diagonal we choose the chiral representation of the gamma matrices,

$$
\gamma^{0}=\left[\begin{array}{cc}
0 & -1 \\
-1 & 0
\end{array}\right], \quad \gamma=\left[\begin{array}{cc}
0 & \boldsymbol{\sigma} \\
-\boldsymbol{\sigma} & 0
\end{array}\right], \quad \gamma^{0} \gamma^{3}=\left[\begin{array}{cc}
\sigma^{3} & 0 \\
0 & -\sigma^{3}
\end{array}\right]
$$

in which,

$$
h(\mathbf{p})=\left[\begin{array}{cc}
\boldsymbol{\sigma} \cdot(\mathbf{p}-e \mathbf{A}) & -m \\
-m & -\boldsymbol{\sigma} \cdot(\mathbf{p}-e \mathbf{A})
\end{array}\right] .
$$

It remains to choose initial conditions for $\chi_{1}$ and $\chi_{2}$ such that the initial condition for $\Omega$ is satisfied. This is most easily done by diagonalizing $\Omega(t=0)$. Let

$$
\Omega(0)=U D U^{\dagger}, \quad \chi_{j}(0)=U \chi_{j}^{D},
$$

for some unitary matrix $U$ chosen in such a way that $D=\operatorname{diag}(1,1,-1,-1)$. Explicitly:

$$
U=\left[\begin{array}{cccc}
\frac{m}{r_{+}} & \frac{-p^{1}+i p^{2}}{r_{+}} & \frac{m}{r_{-}} & \frac{-p^{1}+i p^{2}}{r_{-}} \\
0 & \frac{p^{3}+\omega_{0}}{r_{+}} & 0 & \frac{p^{3}-\omega_{0}}{r_{-}} \\
\frac{p^{3}+\omega_{0}}{r_{+}} & 0 & \frac{p^{3}-\omega_{0}}{r_{-}} & 0 \\
\frac{p^{1}+i p^{2}}{r_{+}} & \frac{m}{r_{+}} & \frac{p^{1}+i p^{2}}{r_{-}} & \frac{m}{r_{-}}
\end{array}\right]
$$

where $r_{+}=\sqrt{2 \omega_{0}\left(\omega_{0}+p^{3}\right)}$ and $r_{-}=\sqrt{2 \omega_{0}\left(\omega_{0}-p^{3}\right)}$. Thus,

$$
\chi_{1}^{D}\left(\chi_{1}^{D}\right)^{\dagger}+\chi_{2}^{D}\left(\chi_{2}^{D}\right)^{\dagger}=1+D=\operatorname{diag}(2,2,0,0)
$$

and we choose,

$$
\chi_{1}^{D}=\sqrt{2}\left[\begin{array}{l}
1 \\
0 \\
0 \\
0
\end{array}\right], \quad \chi_{2}^{D}=\sqrt{2}\left[\begin{array}{l}
0 \\
1 \\
0 \\
0
\end{array}\right]
$$

leading to,

$$
\begin{aligned}
& \chi_{1}(0)=\frac{1}{\sqrt{\omega_{0}\left(\omega_{0}+p^{3}\right)}}\left[\begin{array}{c}
m \\
0 \\
p^{3}+\omega_{0} \\
p^{1}+i p^{2}
\end{array}\right], \\
& \chi_{2}(0)=\frac{1}{\sqrt{\omega_{0}\left(\omega_{0}+p^{3}\right)}}\left[\begin{array}{c}
-p^{1}+i p^{2} \\
p^{3}+\omega_{0} \\
0 \\
m
\end{array}\right] .
\end{aligned}
$$

Now $h^{0}$ is proportional to $\Omega(0), h^{0}=-\omega_{0} \Omega(0)$. This implies that both $\chi_{1}(0)$ and $\chi_{2}(0)$ are eigenspinors of $h^{0}$ with the same eigenvalue $\left(-\omega_{0}\right)$. By $(159)$ we then have,

$$
\partial_{t} \chi_{j}(0)=i \omega_{0} \chi_{j}(0)
$$


Hence we obtain, using (160),

$$
\begin{aligned}
& \chi_{1}=\frac{1}{\sqrt{\bar{\omega}_{0}\left(\bar{\omega}_{0}+\bar{p}^{3}\right)}}\left[\begin{array}{c}
\bar{m} y_{1} \\
0 \\
\left(\bar{p}^{3}+\bar{\omega}_{0}\right) y_{2} \\
\left(\bar{p}^{1}+i \bar{p}^{2}\right) y_{1}
\end{array}\right], \\
& \chi_{2}=\frac{1}{\sqrt{\bar{\omega}_{0}\left(\bar{\omega}_{0}+\bar{p}^{3}\right)}}\left[\begin{array}{c}
\left(-\bar{p}^{1}+i \bar{p}^{2}\right) y_{1} \\
\left(\bar{p}^{3}+\bar{\omega}_{0}\right) y_{2} \\
0 \\
\bar{m} y_{1}
\end{array}\right],
\end{aligned}
$$

where,

$$
\begin{array}{lll}
\ddot{y_{1}}+\left(\bar{\omega}^{2}-i\right) y_{1}=0, & y_{1}(0)=1, & \dot{y}_{1}(0)=i \bar{\omega}_{0}, \\
\ddot{y}_{2}+\left(\bar{\omega}^{2}+i\right) y_{2}=0, & y_{2}(0)=1, & \dot{y}_{2}(0)=i \bar{\omega}_{0},
\end{array}
$$

having introduced dimensionless quantities as for bosons. $y_{1}$ can be written as a linear combination of the (linearly dependent) parabolic cylinder functions,

$$
D_{-\frac{i}{2} \Lambda-1}(-(1+i) \tau), \quad D_{-\frac{i}{2} \Lambda-1}((1+i) \tau), \quad D_{\frac{i}{2} \Lambda}((1-i) \tau), \quad D_{\frac{i}{2} \Lambda}(-(1-i) \tau),
$$

whereas $y_{2}$ can be written in terms of,

$$
D_{-\frac{i}{2} \Lambda}(-(1+i) \tau), \quad D_{-\frac{i}{2} \Lambda}((1+i) \tau), \quad D_{\frac{i}{2} \Lambda-1}((1-i) \tau), \quad D_{\frac{i}{2} \Lambda-1}(-(1-i) \tau)
$$

Note that,

$$
y_{2}=\frac{\left(\bar{p}^{3}-\bar{t}\right) y_{1}-i \dot{y}_{1}}{\bar{\omega}_{0}+\bar{p}^{3}}
$$

\subsection{Expectation value of the number operator}

The number operator, expressed in terms of fields in the $\mathbf{A}=0$ gauge, is simply,

$$
\hat{N}=\int_{p}\left(\frac{1}{2}\left[\hat{\psi}_{\alpha}^{\dagger}(\mathbf{p}), \hat{\psi}_{\beta}(\mathbf{p})\right] \frac{h_{\alpha \beta}^{0}(\mathbf{p})}{\omega_{0}(\mathbf{p})}+2 V\right) .
$$

As for bosons, we transform the covariance to the gauge $\mathbf{A}=0$ by shifting the momentum,

$$
\Omega^{g}\left(\mathbf{p}, t_{f}\right)=\Omega\left(\mathbf{p}+e \mathbf{A}\left(t_{f}\right), t_{f}\right) .
$$

The expectation value of the number operator then follows,

$$
\langle\hat{N}\rangle\left(t_{f}\right)=\operatorname{tr}\left(\hat{N} \hat{\rho}\left(t_{f}\right)\right)=\frac{V}{2} \int_{p}\left(4-\tanh \left(\frac{\beta \omega_{0}^{g}(\mathbf{p})}{2}\right) \operatorname{tr}\left(\Omega(\mathbf{p}, 0) \Omega^{g}\left(\mathbf{p}, t_{f}\right)\right)\right) .
$$

Subtracting the number of particles present at $t=0$,

$$
\left.\langle\hat{N}\rangle\right|_{t=0}=2 V \int_{p}\left(1-\tanh \left(\frac{\beta \omega_{0}}{2}\right)\right)
$$



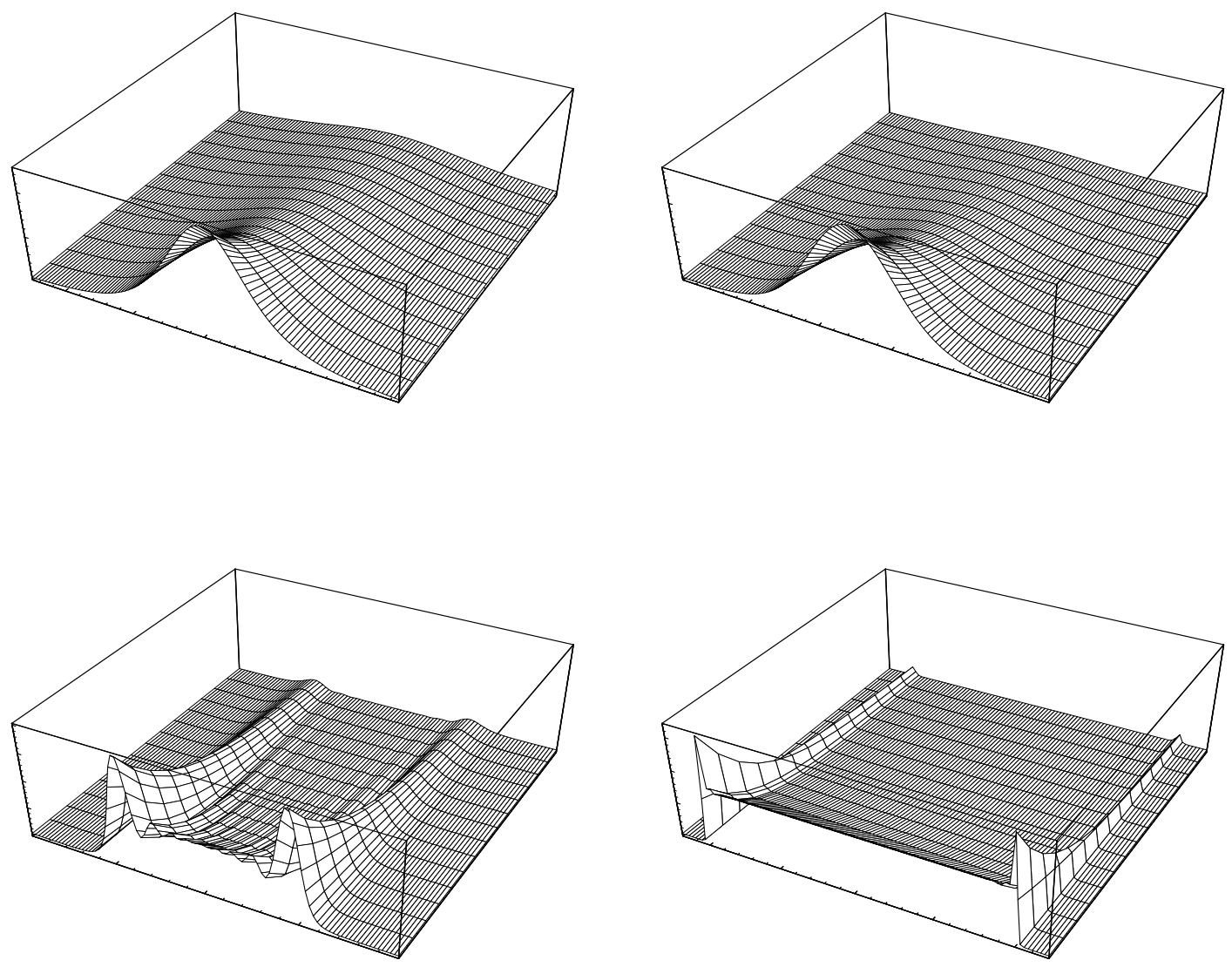

Figure 3: The momentum distribution, $n(-\mathbf{p}) / 2$, of the positrons at zero temperature. All quantities are expressed in units of the electric field.

the number of particles created is,

$$
\langle\hat{N}\rangle^{\mathrm{cr}}\left(t_{f}\right)=\frac{V}{2} \int_{p} \tanh \left(\frac{\beta \omega_{0}^{g}}{2}\right)\left(4-\operatorname{tr}\left(\Omega^{0} \Omega^{g}\right)\right) .
$$

Writing,

$$
\Omega^{0}=\chi_{1}^{0}\left(\chi_{1}^{0}\right)^{\dagger}+\chi_{2}^{0}\left(\chi_{2}^{0}\right)^{\dagger}-1, \quad \Omega^{g}=\chi_{1}^{g}\left(\chi_{1}^{g}\right)^{\dagger}+\chi_{2}^{g}\left(\chi_{2}^{g}\right)^{\dagger}-1
$$

one finds,

$$
\operatorname{tr}\left(\Omega^{0} \Omega^{g}\right)=\sum_{i, j=1}^{2}\left|\left(\chi_{i}^{0}, \chi_{j}^{g}\right)\right|^{2}-4,
$$




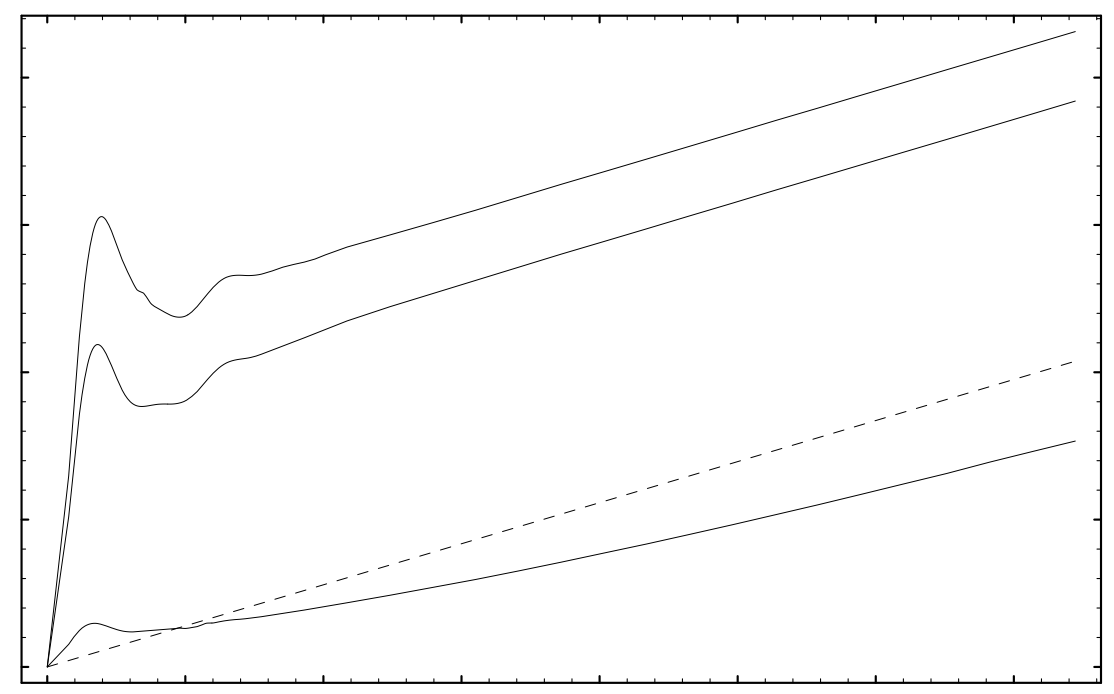

Figure 4: The average number of created fermions for $\bar{m}=1$. The dashed line shows the asymptotic behaviour at zero temperature. All quantities are expressed in units of the electric field.

where $(\chi, \lambda)=\chi_{\alpha} \lambda_{\alpha}^{*}$ and having used that the norm $(\chi, \chi)$ is conserved in time. Using (170) and (171) we obtain,

$$
\sum_{i, j=1}^{2}\left|\left(\chi_{i}^{0}, \chi_{j}^{g}\right)\right|^{2}=2\left|\left(\chi_{1}^{0}, \chi_{1}^{g}\right)\right|^{2}=\frac{2}{\bar{\omega}_{0}\left(\bar{\omega}_{0}+\bar{p}^{3}\right) \bar{\omega}_{0}^{g}\left(\bar{\omega}_{0}^{g}+\bar{p}_{g}^{3}\right)}\left|\Lambda y_{1}^{g}+\left(\bar{\omega}_{0}+\bar{p}^{3}\right)\left(\bar{\omega}_{0}^{g}+\bar{p}_{g}^{3}\right) y_{2}^{g}\right|^{2} .
$$

Finally, writing $y_{1}=r e^{i \theta}$ and again using the conservation of the spinor norm, we find

$$
\langle\hat{N}\rangle^{\mathrm{cr}}\left(t_{f}\right)=V \int_{p} n\left(\mathbf{p}, t_{f}\right) \tanh \left(\frac{\bar{\beta} \bar{\omega}_{0}^{g}}{2}\right),
$$

having defined the zero temperature number density $n\left(\mathbf{p}, t_{f}\right)$ as

$$
n\left(\mathbf{p}, t_{f}\right)=2\left[1-\frac{\bar{p}^{3}}{\bar{\omega}_{0}}-\left(1-\frac{\bar{p}_{g}^{3}}{\bar{\omega}_{0}^{g}}\right) \frac{l_{g}}{\bar{\omega}_{0}}\right]
$$

where

$$
\begin{aligned}
\ddot{r}-\frac{l^{2}}{r^{3}}+\bar{\omega}^{2} r & =0, \quad r(0)=1, \quad \dot{r}(0)=0, \\
i & =r^{2}, \quad l(0)=\bar{\omega}_{0}, \\
\dot{\theta} & =\frac{l}{r^{2}}, \quad \theta(0)=0 .
\end{aligned}
$$

The subscript (superscript) $g$ still indicates gauge transformed quantities i.e. $\bar{p}^{3}$ is shifted, $\bar{p}_{g}^{3}=\bar{p}^{3}+\bar{t}_{f}$. Figure 3 shows $n\left(-\mathbf{p}, t_{f}\right) / 2$, the average momentum distribution of the 
created positrons at zero temperature. Comparing with the corresponding result for bosons, figure 1, there is an interesting difference for small times. Lots of fermions are created with zero momentum while hardly any bosons are. The result of numerically evaluating the integral (185) with a mass $\bar{m}=1$ for some different temperatures is shown in figure 4 . Increasing the temperature clearly decreases the particle production but again, as for bosons, for large enough times the slope is independent of temperature. This suppression of particle production can be understood in a simple model at fixed spin and momentum. We then have four states: $|0\rangle,\left|e^{-}\right\rangle,\left|e^{+}\right\rangle$and $\left|e^{-} e^{+}\right\rangle$. Due to charge conservation the only allowed particle production- and annihilation processes are: $|0\rangle \rightarrow$ $\left|e^{-} e^{+}\right\rangle,\left|e^{-} e^{+}\right\rangle \rightarrow|0\rangle$. At infinite temperature the probabilities for $|0\rangle$ and $\left|e^{-} e^{+}\right\rangle$ are equal in the initial ensemble. Hence creation and annihilation processes are equally probable leading to no creation on the average.

If we have an electric field with an arbitrary time dependence as for bosons, (81), we have to change $\bar{\omega}$ accordingly, gauge transformations become $\bar{p}_{g}^{3}=\bar{p}^{3}+F\left(\bar{t}_{f}\right)$ and we also have to change (188) into $i=f(\bar{t}) r^{2}$.

\subsection{Asymptotic expansion}

Let us now examine what happens to the number density $n\left(\Lambda, \bar{p}^{3}, \bar{t}_{f}\right)$ when $\bar{t}_{f}$ is large, $\bar{t}_{f}>>1$. As for bosons, consider $\Lambda$ and $\bar{p}^{3}$ satisfying,

$$
\sqrt{\Lambda}<<\bar{t}_{f}, \quad \sqrt{\Lambda}-\bar{t}_{f}<<\bar{p}^{3}<<-\sqrt{\Lambda}, \quad \bar{p}^{3}<<-1 .
$$

The initial condition for $\Omega^{g}$ is,

$$
\Omega^{g}(\mathbf{p}, 0)=-\gamma^{0} \frac{\left(\boldsymbol{\gamma} \cdot\left(\mathbf{p}+e \mathbf{A}\left(t_{f}\right)\right)+m\right)}{\omega_{0}\left(\mathbf{p}+e \mathbf{A}\left(t_{f}\right)\right)} .
$$

Using (90) one finds $\Omega^{g}(\mathbf{p}, t)$ for $\mathbf{p}$ restricted by (190) as,

$$
\begin{aligned}
& \chi_{1}^{g}(\bar{t})=\sqrt{2} e^{\frac{-\pi \Lambda}{8}} {\left[\begin{array}{l}
\frac{1+i}{2} \bar{m} D_{-\frac{i}{2} \Lambda-1}\left(-(1+i)\left(\bar{t}-\bar{t}_{f}-\bar{p}^{3}\right)\right) \\
0 \\
D_{-i} \Lambda \\
\frac{1+i}{2}\left(\bar{p}^{1}+i \bar{p}^{2}\right) D_{-\frac{i}{2} \Lambda-1}\left(-(1+i)\left(\bar{t}-\bar{t}_{f}-\bar{p}^{3}\right)\right)
\end{array}\right], } \\
& \chi_{2}^{g}(\bar{t})=\sqrt{2} e^{\frac{-\pi \Lambda}{8}}\left[\begin{array}{l}
\frac{1+i}{2}\left(-\bar{p}^{1}+i \bar{p}^{2}\right) D_{-\frac{i}{2} \Lambda-1}\left(-(1+i)\left(\bar{t}-\bar{t}_{f}-\bar{p}^{3}\right)\right) \\
D_{-\frac{i}{2} \Lambda}\left(-(1+i)\left(\bar{t}-\bar{t}_{f}-\bar{p}^{3}\right)\right) \\
0 \\
\frac{1+i}{2} \bar{m} D_{-\frac{i}{2} \Lambda-1}\left(-(1+i)\left(\bar{t}-\bar{t}_{f}-\bar{p}^{3}\right)\right)
\end{array}\right] .
\end{aligned}
$$

Note that the $\chi_{j}^{g}$ here are not normalized exactly in the same way as in (167) and (168), the normalization differs by some irrelevant phases. Again for $\mathbf{p}$ restricted by (190), we obtain, using (91),

$$
n\left(\Lambda, \bar{p}^{3}, \bar{t}_{f}\right)=2-\frac{1}{2} \operatorname{tr}\left(\Omega^{0} \Omega^{g}\left(t_{f}\right)\right)=4 e^{-\pi \Lambda}
$$


Thus, using a similar reasoning as for bosons, we conclude that for $\bar{t}_{f}>>1$ and $\bar{t}_{f}>>\bar{m}$,

$$
n\left(\Lambda, \bar{p}^{3}, \bar{t}_{f}\right)= \begin{cases}4 e^{-\pi \Lambda}, & -\bar{t}_{f}<\bar{p}^{3}<0 \\ 0, & \text { otherwise }\end{cases}
$$

Comparing to the analogous expression for bosons, (93), we note that the only difference is a factor of 2 arising from the spin degrees of freedom of the fermions. Accordingly we obtain

$$
\langle\hat{N}\rangle^{\mathrm{cr}}\left(\bar{t}_{f}\right)=\frac{\bar{V}}{2(2 \pi)^{2}} \int_{-\bar{t}_{f}}^{0} d \bar{p}^{3} \int_{\bar{m}^{2}}^{\infty} d \Lambda 4 \tanh \left(\frac{\bar{\beta} \bar{\omega}_{0}^{g}}{2}\right) e^{-\pi \Lambda}, \quad \bar{t}_{f}>>1, \quad \bar{t}_{f}>>\bar{m} .
$$

Considering the limits $\frac{1}{\beta}<<\bar{t}_{f}$ and $\frac{1}{\beta}>>\bar{t}_{f}$ of $(\sqrt{196})$, we find

$$
\begin{aligned}
\langle\hat{N}\rangle^{\mathrm{cr}}\left(\bar{t}_{f}\right) & =\frac{4 \bar{V}}{(2 \pi)^{3}} \bar{t}_{f} e^{-\pi \bar{m}^{2}}=\frac{2 \alpha}{\pi^{2}} V E^{2} t_{f} e^{-\frac{\pi m^{2}}{|e E|}}, \quad \frac{1}{\bar{\beta}}<<\bar{t}_{f}, \\
\langle\hat{N}\rangle^{\mathrm{cr}}\left(\bar{t}_{f}\right) & =\frac{\bar{\beta} \bar{V}}{(2 \pi)^{3}} \bar{t}_{f}^{2} e^{-\pi \bar{m}^{2}}=\left(\frac{\alpha}{\pi}\right)^{3 / 2} \beta V|E|^{3} t_{f}^{2} e^{-\frac{\pi m^{2}}{e E \mid}}, \quad \frac{1}{\bar{\beta}}>>\bar{t}_{f} .
\end{aligned}
$$

\subsection{Probabilities}

We will now as for bosons give the expressions for the probability $P\left(n, m ; t_{f}\right)$ of finding $n$ electrons and $m$ positrons in the ensemble at time $t=t_{f}$. The calculations are however a good deal more involved for fermions so we will illustrate what happens by calculating $P\left(0,0 ; t_{f}\right)$ explicitly. A particular choice of groundstate $\Psi_{0}$ is, (remember that the representation is reducible),

$$
\Psi_{0}\left(\eta^{*}, \eta\right)=\operatorname{det}\left(\frac{I}{\sqrt{2}}\right) \exp \left(\int_{p} \eta^{*} \Omega^{0} \eta\right)
$$

We find in momentum space

$$
\begin{aligned}
& \left\langle\Psi_{0}\left|\hat{\rho}\left(t_{f}\right)\right| \Psi_{0}\right\rangle= \\
& =\operatorname{det}\left(\frac{I}{4}\right) \operatorname{det}\left(\Omega^{0}+\Omega^{\beta}\right) \times \\
& \quad \operatorname{det}\left(\left(\Omega^{\beta}-1\right)\left(\Omega^{0}+\Omega^{\beta}\right)\left(\Omega^{\beta}-1\right)^{-1}-\left(\Omega^{\beta}-1\right)\left(\Omega^{\beta}+1\right)\left(\Omega^{0}+\Omega^{\beta}\right)^{-1}\right) \\
& =\operatorname{det}\left[\operatorname{det}_{s p}\left(\frac{\left(\Omega^{\beta}-1\right)\left(\Omega^{0}+\Omega^{\beta}\right)\left(\Omega^{\beta}+1\right)\left(\Omega^{0}+\Omega^{\beta}\right)}{4\left(t h^{2}-1\right)}+\frac{1-t h^{2}}{4}\right)\right]
\end{aligned}
$$

where $\Omega^{\beta}=t h \Omega^{g}\left(t_{f}\right)$, th $=\tanh \left(\frac{\bar{\beta} \bar{\omega}_{0}^{g}}{2}\right)$ and $\operatorname{det}_{s p}$ denotes the determinant evaluated only with respect to the spinor indices. Now express $\Omega^{0}$ and $\Omega^{g}\left(t_{f}\right)$ in terms of spinors, see (182), and use

$$
\operatorname{det}_{s p}(A)=\frac{1}{4 !}\left[(\operatorname{tr} A)^{4}-6(\operatorname{tr} A)^{2}\left(\operatorname{tr} A^{2}\right)+8 \operatorname{tr} A \operatorname{tr} A^{3}+3\left(\operatorname{tr} A^{2}\right)^{2}-6 \operatorname{tr} A^{4}\right],
$$


which holds for a $4 \times 4$-matrix $A$. Using a computer to handle the algebra we obtain

$$
\begin{aligned}
P\left(0,0 ; t_{f}\right) & =\left\langle\Psi_{0}\left|\hat{\rho}\left(t_{f}\right)\right| \Psi_{0}\right\rangle=\operatorname{det}\left(\frac{1}{16}\left(1+t h\left(\left|\left(\chi_{1}^{0}, \chi_{1}^{g}\right)\right|^{2}-2\right)+t h^{2}\right)^{2}\right) \\
& =\exp \left(2 V \int_{p} \ln \left[\frac{1}{4}\left(1+t h\left(2-n\left(t_{f}\right)\right)+t h^{2}\right)\right]\right),
\end{aligned}
$$

expressed in terms of the zero temperature number density $n\left(t_{f}\right)$ defined in (186) . At zero temperature this reduces to

$$
P\left(0,0 ; t_{f}\right)=\exp \left[2 \operatorname{Tr} \ln \left(1-\frac{n\left(t_{f}\right)}{4}\right)\right],
$$

which for large times, $\bar{t}_{f}>>1$, by (195) becomes

$$
P\left(0,0 ; t_{f}\right)=\exp \left[\frac{\bar{V} \bar{t}_{f}}{(2 \pi)^{2}} \int_{\bar{m}^{2}}^{\infty} d \Lambda \ln \left(1-e^{-\pi \Lambda}\right)\right] .
$$

Again this result agrees with the result of Schwinger [1]. In general we express the probability $P\left(n, m ; t_{f}\right)$ in terms of a generating function $f\left(x, y ; t_{f}\right)$ as for bosons, (100),

$$
f\left(x, y ; t_{f}\right)=\exp \left(2 \operatorname{Tr} \ln \left[\left(1+x g\left(t_{f}\right)\right)\left(1+y g\left(t_{f}\right)\right)+x y h\left(t_{f}\right)\right]\right)
$$

where $g\left(t_{f}\right)$ and $h\left(t_{f}\right)$ are

$$
\begin{aligned}
g\left(t_{f}\right) & =\frac{2}{\left(2-n\left(t_{f}\right)+2 \operatorname{coth} \bar{\beta} \bar{\omega}_{0}^{g}\right) \sinh \bar{\beta} \bar{\omega}_{0}^{g}}, \\
h\left(t_{f}\right) & =\frac{n\left(t_{f}\right)\left(4-n\left(t_{f}\right)\right)}{\left(2-n\left(t_{f}\right)+2 \operatorname{coth} \bar{\beta} \bar{\omega}_{0}^{g}\right)^{2}} .
\end{aligned}
$$

Finally calculating the expectation value of the number operator

$$
\begin{aligned}
\langle\hat{N}\rangle\left(t_{f}\right) & =\sum_{n, m}^{\infty}(n+m) P(n, m)=\left.f(1,1)^{-1} \partial_{x} f(x, x)\right|_{x=1} \\
& =\operatorname{Tr}\left(2+\tanh \left(\frac{\bar{\beta} \bar{\omega}_{0}^{g}}{2}\right)\left(n\left(t_{f}\right)-2\right)\right),
\end{aligned}
$$

which agrees with $(179)$.

\section{Final remarks}

We will briefly comment on the infinite time limit of $P\left(0,0 ; t_{f}\right)$ since this quantity is usually discussed. We will do this for fermions. For bosons, a similar argument may be made. The expression (202) for $P\left(0,0, t_{f}\right)$ may be rewritten when $\bar{t}_{f}>>1$. Using (195) we find for an arbitrary temperature

$$
P\left(0,0 ; t_{f}\right)=\exp \left[C(\bar{\beta})+\frac{2 \bar{V}}{2(2 \pi)^{2}} \int_{\bar{m}^{2}}^{\infty} d \Lambda \int_{-\bar{t}_{f}}^{0} d \bar{p}^{3} \ln \frac{1+t h\left(2-4 e^{-\pi \Lambda}\right)+t h^{2}}{(1+t h)^{2}}\right]
$$


where $C(\bar{\beta})$ is a time independent constant and $t h=\tanh \left(\frac{\bar{\beta} \bar{\omega}_{0}^{g}}{2}\right)$. The only time dependence is in $\bar{\omega}_{0}^{g}\left(t_{f}\right)$. From this expression it is clear that $w$, in the literature misleadingly known as the pair production rate, is

$$
w=\lim _{t_{f} \rightarrow \infty} \frac{\ln P\left(0,0 ; t_{f}\right)}{V t_{f}}=\frac{\alpha E^{2}}{\pi} \int_{\bar{m}^{2}}^{\infty} d \Lambda \ln \left(1-e^{-\pi \Lambda}\right)
$$

for any temperature. Therefore in the strict infinite time limit $w$ is independent of temperature, even though for intermediate times it certainly does depend on temperature. However, the quantity that is directly related to the observable particle production is not $w$ but the average of the number operator, $\langle\hat{N}\rangle^{\mathrm{cr}}$, which we have thoroughly discussed.

\section{Acknowledgements}

We wish to thank Per Sundell for some help in the early stages of this work, Per Salomonsson for a stimulating discussion and especially Bo-Sture Skagerstam for many interesting remarks and useful comments throughout this work.

\section{References}

[1] J. Schwinger, On gauge invariance and vacuum polarization, Phys. Rev. 82 (1951) 664.

[2] R. Parentani and R. Brout, Vacuum Instability and black hole radiation, Nucl. Phys. B 388 (1992) 474.

[3] K. Sailer et al, Strings and ropes in heavy ion collisions, J. Phys. G: Nucl. Part. Phys. 17 (1991) 1005.

[4] R.-C. Wang and C.-Y. Wong, Finite-size effects in the Schwinger particle-production mechanism, Phys. Rev. D 38 (1988) 348.

[5] Y. Kluger, J.M. Eisenberg, B. Svetitsky, F. Cooper and E. Mottola: Fermion pair production in a strong electric field, Phys. Rev. D 45 (1992) 4659; Pair production in a strong electric field, Phys. Rev. Lett. 67 (1991) 2427; Particle production in the central rapidity region, Phys. Rev. D 48 (1993) 190.

F. Cooper and E. Mottola, Quantum back reaction in scalar QED as an initial value problem, Phys. Rev. D 40 (1989) 456.

[6] P. Elmfors and B.-S. Skagerstam, Electromagnetic fields in a thermal background, NORDITA-94/18 P (hep-ph/9404106), preprint.

[7] M. Loewe and J.C. Rojas, Thermal effects and the effective action of quantum electrodynamics, Phys. Rev. D 46 (1992) 2689. 
[8] P.H. Cox, W.S. Hellman and A. Yildiz, Finite temperature corrections to field theory: electron mass, magnetic moment and vacuum energy, Ann. Phys. (N.Y.) 154 (1984) 211.

[9] R. Floreanini and R. Jackiw, Functional representation for fermionic quantum fileds, Phys. Rev. D 37 (1988) 2206.

[10] C. Kiefer, Functional Schrödinger equation for scalar QED, Phys. Rev. D 45 (1992) 2044.

[11] C. Kiefer and A. Wipf, Functional Schrödinger equation for fermions in external gauge fields, Ann. Phys. (N.Y.) 236 (1994) 241.

[12] R.P. Feynman, Statistical Mechanics, Frontiers in Physics (1972).

[13] I.S. Gradshteyn and I.M. Ryzhik, Table of integrals, series and products, Academic, London (1980), pp. 1064-1067

[14] E.S. Fradkin, D.M. Gitman and S.M. Shvartsman, Quantum Electrodynamics with unstable vacuum, Springer-Verlag. Includes an extensive reference list on the subject. 